\title{
1 Stabilizing selection shapes variation in phenotypic plasticity
}

5 Dörthe Becker ${ }^{1,2,{ }^{*} \text {, }}$

6 Karen Barnard-Kubow ${ }^{1,3}$,

7 Robert Porter ${ }^{1}$,

8 Austin Edwards ${ }^{1,4}$,

9 Erin Voss ${ }^{1,5}$,

10 Andrew P. Beckerman²,

11 Alan O. Bergland ${ }^{1, *}$

14 1Department of Biology; University of Virginia, Charlottesville/VA, USA

15 2Department of Animal and Plant Sciences; University of Sheffield, Sheffield, UK

$16{ }^{3}$ Department of Biology, James Madison University, Harrisonburg/VA, USA

17 4Biological Imaging Development CoLab, University of California San Francisco,

18 San Francisco/CA, USA

19 Department of Integrative Biology, University of California Berkeley,

20 Berkeley/CA

22 * to whom correspondence should be addressed:

23 DB (d.becker@sheffield.ac.uk), AOB (aob2x@virginia.edu)

27 Keywords: stabilizing selection; phenotypic plasticity; heritability; Daphnia;

28 defense morphologies 


\section{Abstract}

30 The adaptive nature of phenotypic plasticity is widely documented in natural

31 populations. However, little is known about the evolutionary forces that shape

32 genetic variation in plasticity within populations. Here we empirically address this

33 issue by testing the hypothesis that stabilizing selection shapes genetic variation

34 in the anti-predator developmental plasticity of Daphnia pulex. The anti-predator

35 morphological defense is characterized by pedestal and spikes that grow on the

36 back of the Daphnia neck following exposure to predator cure. We characterized

37 variation in this plasticity using a novel, high-throughput phenotyping method that

38 describes the entire dorsal shape amongst $>100$ D. pulex strains originating from

39 a natural population in the UK. We found low genetic diversity for morphological

40 defenses among genetically diverse clones upon predation risk exposure. The

41 strongest reduction in genetic variation was observed in areas of greatest

42 phenotypic plasticity, which we interpret as evidence of stabilizing selection. By

43 assessing among-clone variance in clonally related, field derived strains, we

44 contrasted mutational variation $\left(V_{m}\right)$ to standing variation $\left(V_{g}\right)$. We found that $V_{g} / V_{m}$

45 is lowest in areas of greatest plasticity. These data strongly suggest that stabilizing

46 selection operates directly on phenotypic plasticity, providing a rare glimpse into

47 the evolution of fitness related traits in natural populations. 


\section{Introduction}

51 Organisms frequently experience temporal and spatial fluctuations in their

52 natural habitats. The capacity to persist and thrive across variable environmental

53 conditions is due to local adaptation at the population level, and phenotypic

54 plasticity at the individual level. Phenotypic plasticity, the ability of a single

55 genotype to alter its phenotype in response to environmental change, is ubiquitous

56 in natural populations and well established as an adaptive response to

57 environmental change [1, 2]. Providing time for a population to become

58 established, adaptive plasticity reduces the probability of extinction in new or

59 fluctuating environments [3, 4], and also enables populations to efficiently traverse

60 fitness landscapes [3]. Decades of theoretical and empirical work on the

61 quantitative genetics of plasticity has revealed that genetic variation for plasticity

62 exists $[5,6]$, that heritable variation in plasticity can respond to natural selection [1,

63 7] and that plasticity is itself locally adapted (e.g., [8]).

64 Evidence for the adaptive nature of phenotypic plasticity has been

65 demonstrated for morphological and life-history traits [9]. Broadly, this work is

66 aligned with theoretical predictions that adaptive phenotypic plasticity evolves

67 when environmental change is rapid [10,11], environmental cues are reliable [12,

68 13], plastic responses occur as rapidly as environmental change [14], and when

69 the incurred cost of plasticity is low [15, 16]. Empirical studies on the adaptive

70 nature of phenotypic plasticity often measure aspects of fitness following

71 environmental exposures (e.g., [17]), or document the plastic responses of 
72 genotypes collected from different locations that reflect alternate historical

73 selection regimes (e.g., [18]).

74 However, such data and insights do not reveal the selective forces shaping

75 levels of genetically based phenotypic variation within populations. Theoretical

76 models aimed at explaining the level of quantitative genetic variation in natural

77 populations frequently highlight the central role of stabilizing selection [19, 20].

78 Stabilizing selection reduces functional genetic variation in a population without

79 modifying the population mean and maintains populations near their local fitness

80 peak. Yet there is a stark gap between the theoretical expectation that stabilizing

81 selection commonly operates on natural populations and the empirical evaluation

82 of stabilizing selection [21, 22] (but see [23, 24]). This data deficit is especially

83 pronounced for phenotypic plasticity, because of the compounding challenges of

84 measuring genetic variation in plasticity (e.g., $[12,25])$ and of estimating mutational

85 variation, which is required to test hypotheses about the mechanisms that shape

86 the magnitude of genetically based phenotypic diversity [25, 26].

87 We directly address this gap by evaluating the signature of stabilizing selection

88 on predator induced defense in the eco-evolutionary model, Daphnia pulex. This

89 species can develop a defensive, spikey pedestal when in the presence of

90 predatory Chaoborus spp. larvae and is a century old, classic example of adaptive

91 phenotypic plasticity $[27,28]$. The predator induced defenses are size and age

92 specific and lead to an increased survival to predator attack by up to $50 \%[28,29]$.

93 Induction in the absence of actual predators incurs a fitness cost [28, 30]. 
94 We combine a novel, high-throughput phenotyping assay with genome re-

95 sequencing to test the hypothesis that this plastic response is subject to stabilizing

96 selection. We first show that genetic variation for the defense, in an outbred

97 population is reduced upon exposure to predator cue. The reduction of genetic

98 variation is greatest in the region of the dorsal axis with greatest plasticity. We

99 demonstrate that this region is an independent phenotypic module, suggesting that

100 the pleiotropic costs to plasticity may have been minimized. To evaluate the

101 signature of stabilizing selection, we measured mutational variation of plasticity

102 using clonally related strains, and contrasted mutational variation $\left(\mathrm{V}_{\mathrm{m}}\right)$ to standing

103 variation $\left(\mathrm{V}_{\mathrm{g}}\right)$ in the outbred individuals. Consistent with the view that plasticity is

104 subject to stabilizing selection, we show that $\mathrm{V}_{\mathrm{g}} / \mathrm{V}_{\mathrm{m}}$ is lowest in the region of

105 greatest plasticity. Taken together, these data provide unique insight into the

106 evolution of phenotypic plasticity and the forces that shape genetic variation in the

107 wild.

108

\section{Results}

111 Robust and accurate phenotyping. To date, assessment of predator induced

112 morphological changes in Daphnia have been based on a categorical scoring

113 technique that classifies pedestals as absent (score $=0)$, small (score $=30$ ) or

114 large (score $=50$ ) and adds spikes in increments of $10[28,29]$. Although this

115 scoring technique has been useful, its coarse scale and potential for observer bias

116 limits its capacity to assess plasticity with high resolution, high replication and 
117 reproducibility, and low observer bias.

118 To remedy this, we developed a novel analysis tool, DAPCHA, that allows

119 automated identification of defined phenotypic landmarks and subsequent

120 quantification of phenotypic plasticity using standardized photographic images

121 from stereoscope microscopy (Fig. 1A). These features include basic aspects of

122 morphology such as animal length, eye size, tail length (Fig. 1A-II), as well as a

123 trace of the entire dorsal edge (Fig. 1A-III). DAPCHA accurately measures animal

124 length (Fig. 1B; Suppl. Tab. 1) and is more reliable in unit conversion of length

125 estimates than are manual observers (e.g., run 2, Fig. 1C; Suppl. Tab. 1).

126 We used DAPCHA to calculate the maximum dorsal height of the animal (Fig.

$1271 \mathrm{~A}-\mathrm{V}$, and see Materials and Methods), as a summary of morphological plasticity

128 before and after exposure to predator derived kairomones. We find that maximum

129 dorsal height significantly changes with the production of pedestals and spikes in

130 response to predator cue (Fig. 1D; Suppl. Tab. 1) and is also strongly correlated

131 with manual assessments based on the discrete manual scale, described above

132 (Suppl. Tab. 1). We also show that there was inconsistent manual assessment of

133 the pedestal score between three independent observers (Fig. 1D; Suppl. Tab. 1),

134 suggesting that $D A P C H A$ is a useful approach to assess plasticity in an unbiased

135 manner. We retroactively assigned animals to the first instar and second instar

136 (Fig. 1E) based on the multimodal distribution of animal length and animal dorsal

137 area (see Materials and Methods), enabling us to ask questions about the extent

138 of plasticity in distinct size and age classes. 
140 Evidence for stabilizing selection in an outbred sample. We estimated components

141 of phenotypic variation in the plastic response among 49 genetically unique strains

142 (Suppl. Fig. 1) sampled from a single population in southern England ([31]). We

143 reared each strain with and without kairomone and photographed ca. 4 individuals

144 per strain and treatment group each day, for the first 3-4 days post-parturition (471

145 individuals, Suppl. Fig. 2).

146 First, we evaluated the basic plastic response of these strains. We

147 demonstrate that exposure to kairomone induces a plastic response, most

148 prominently in the nuchal area (Fig. 2A,E; Suppl. Fig. 3). The magnitude and

149 position of the defense varied with predation risk. The maximum dorsal height

150 shifted towards anterior head regions (Fig. 2A,B; Suppl. Fig. 3; Suppl. Tab. 1) and

151 the maximum height of the defense structures increased (Fig. 2C; Suppl. Tab. 1).

152 Furthermore, we detected an increase in the number of neckteeth under predation

153 risk (Fig. 2D; Suppl. Tab. 1). A quantitative genetic test of modularity (see Materials

154 and Methods) indicates that standing genetic variation in the region of maximum

155 plasticity is uncorrelated with the rest of the anterior-posterior (A-P) axis,

156 suggesting that this region responds to selection as an independent phenotypic

157 module (Suppl. Fig. 4). Such a pattern suggests that pleiotropic costs to plasticity

158 may have been minimized.

159 We decomposed phenotypic variation in plasticity among these individuals to

160 estimate the relative contribution of genotype, environment, and genotype-by-

161 environment at each position along the dorsal A-P axis. We observe a strong

162 plastic response of dorsal height among our outbred population (Fig. 2E, red line) 
163 and, as expected, the peak of induction is centered in the nuchal area around

164 dorsal position 150. We observed substantial genetic variation in the induced

165 morphological defense (Fig. 2E, blue line).

166 There is a pronounced increase in GxE variation for instar 2 around position

167150 (Fig. 2E, grey line). This elevated GxE near the region of maximum induction

168 could be caused by crossing reaction norms or by a change in genetic variance

169 between the control and predator cue environments. To test these alternative

170 explanations, we calculated both the broad- and narrow-sense heritability of dorsal

171 height in the two environments. We find a reduction of both measures of heritability

172 for dorsal height upon exposure to predator cue slightly dorsal to the region of

173 maximum plasticity (Fig. 2F,G; Suppl. Tab. 1). This result is consistent with the

174 hypothesis that phenotypic plasticity is subject to stabilizing selection.

175

176 Mutational variation and further evidence of stabilizing selection. We contrasted

177 levels of standing genetic variation, $V_{\mathrm{g}}$, with mutational variation, $\mathrm{V}_{\mathrm{m}}$, as an

178 additional test for stabilizing selection [32]. To estimate mutational variation in

179 phenotypic plasticity, we characterized 56 clonally related strains (Suppl. Fig. 1)

180 using the same experimental design as described above (516 individuals) and

181 assayed concurrently with the genetically unique clones. These clonally related

182 strains were independently isolated from the field and sampled from the same

183 ponds at the same time as the outbred individuals, above, allowing us to directly

184 relate levels of genetic variation in the plastic response. Population genomic 
185 analysis suggests that these clonally related isolates share a recent common

186 ancestor and are also related to the outbred individuals that we studied here ([31]).

187 Detailed assessment of the plastic response of these clonally related strains

188 and their among-line variance reveal that these clonally related strains display a

189 robust plastic response that is similar to the average plastic response of the

190 outbred individuals (Suppl. Fig. 5). We also detected considerable variation in

191 plasticity among these clonally related strains in several key metrics of plasticity

192 such as the height along the A-P axis (Suppl. Fig. 5A; Suppl. Tab. 1), and the

193 maximum height of the pedestal (Suppl. Fig. 5C; Suppl. Tab. 1).

194 We confirmed that the phenotypic variance that we observe among these

195 clonally related strains is heritable, and unlikely to be caused by other factors such

196 as maternal effects or experimental artifact, by performing a 'twin analysis'. For

197 this analysis, we took advantage of a feature of our experimental design,

198 evaluating the correlation of plasticity between siblings released from the same

199 mother ('within clutch'), and between individuals from the same strain but born to

200 different mothers ('within clone'), replicated across the 56 strains of the same

201 clonal assemblage. If the significant among-line variance that we observe across

202 the clonally related strains were due to experimental artifact or shared

203 environment, the correlation between individuals released from the same mother

204 ('twins'; within clutch) should be higher than the correlation between individuals

205 released from different mothers of the same strain ('cousins'; within clone).

206 Similarly, if the among-line variance of the clonally related strains is due

207 experimental artifact, the correlation between individuals within a batch should be 
208 high. On the other hand, if new mutations (or heritable epigenetic marks, see e.g.,

209 [33]) cause phenotypic differentiation between clonally related strains, then twins

210 should be (i) as similar to each other as cousins, and (ii) should be more similar to

211 each other than to other genetically similar lineage of the clonal assemblage

212 (among clones).

213 In line with the expectation that mutations underlie variation, we detected strong

214 correlation between twins and between cousins and that these individuals are

215 more similar to each other than to randomly paired individuals sampled across the

216 clonal assemblage (Fig. 3A; Suppl. Tab. 1). Moreover, the correlation coefficients

217 between twins broadly exceeded the expectations from permutations (Fig. 3A,

218 'within clutch'; Suppl. Tab. 1), while correlations of randomly paired individuals

219 from the clonal assemblage were markedly lower (Fig. 3A, 'within clutch' and

220 'among clones'; Suppl. Tab. 1). We also detected no considerable correlation of

221 phenotypic responses among individuals from the same experimental batches

222 (Fig. 3A, 'among batches'; Suppl. Tab. 1), suggesting that both experimental batch

223 and maternal effects are not driving the observed variance among clonally related

224 strains.

225 From this analysis, plus the linear model approach described above, we

226 conclude that there are heritable differences in plasticity between strains that are

227 clonally related. We interpret this among-line variance as mutational variance, $\mathrm{V}_{\mathrm{m}}$.

228 The magnitude of $V_{m}$ that we document here is on par with estimates of mutational

229 variance in other Daphnia studies [34]. The large mutational variance also

230 demonstrates that plasticity is not mutation limited and suggests that local 
231 adaptation in the plastic response (e.g., [8]) could, in principle, be driven by new

232 mutations that arise frequently.

233 Under a model of mutation-selection balance, which assumes that mutations

234 are either neutral or mildly deleterious, the strength of stabilizing selection on

235 quantitative traits can be estimated from the ratio of standing genetic variation and

236 the mutational variance [35]. We used the among line variance of genetically

237 unique strains as an estimate of $V_{g}$ and the among line variance of clonally related

238 strains as an estimate of $\mathrm{V}_{\mathrm{m}}$. We detected that $\mathrm{V}_{\mathrm{g}} \mathrm{V}_{\mathrm{m}}$ is lowest in areas of largest

239 plasticity around dorsal positions 100 to 250 , in second instar animals exposed to

240 predation risk (Fig. 3B). The localized reduction of $V_{g} / V_{m}$, coupled with the

241 reduction of $V_{g}$ in the induced state (Fig. 2F,G), provides strong evidence for

242 stabilizing selection operating on plasticity.

\section{Discussion}

246 We tested the hypothesis that a classic example of phenotypic plasticity -

247 predator induced defense in D. pulex - is subject to stabilizing selection. We

248 provide two lines of evidence to support our hypothesis: we show that standing

249 genetic variation is reduced in regions of greatest plasticity (Fig. 2) and we show

250 that standing genetic variation in plasticity is substantially lower than mutational

251 variation (Fig. 3). Although the intuitive model that plasticity is directly related to

252 fitness forms the basis for a century of work studying the anti-predator responses

253 of $D$. pulex $[28,36]$ and other related Cladocera [37, 38], to our knowledge, this 
254 hypothesis has not been directly tested. Our work, therefore, provides novel insight

255 into the evolutionary history of phenotypic plasticity.

256 Prior research has demonstrated that aspects of phenotypic plasticity can

257 evolve in response to changes in the environment $[3,4]$. Theoretical models that

258 explain the evolution of plasticity generally assume that genetic diversity is

259 sufficiently present [39, 40], and this assumption is generally realized when

260 examining empirical data in a wide variety of species (e.g., [41]). Despite the

261 ubiquity of genetic variation in plasticity across the tree of life, and genetically

262 based phenotypic variation of fitness-related traits in general [22], determining the

263 evolutionary forces that act on that variation remains a fundamental challenge [42].

264 Determining the evolutionary history of phenotypic variation requires a

265 comparative approach, and often comparisons are made between populations to

266 study local adaptation $[8,17]$ or across taxa to study diversification $[43,44]$. As a

267 consequence, identifying the forces that shape genetically based phenotypic

268 variation within populations remains relatively understudied.

269 In order to gain insight into the evolutionary determinants of phenotypic

270 variation within populations, it is critical to understand the extent of mutational

271 variance. Mutational variance is generally considered deleterious [45], and

272 therefore the rate at which it is removed from populations should reflect the

273 strength of purifying selection [46]. Although directly observing the trajectory of

274 new mutations is challenging, comparisons of standing genetic variation to

275 mutational variation can yield insight into the expected persistence time of new,

276 deleterious mutations [46], the mutational target size [47] and the genetic 
277 architecture of different classes of traits [34]. For instance, the values of $V_{g} / V_{m}$ that

278 we observe are consistent with strong stabilizing selection which removes

279 deleterious mutations quickly from the population via natural selection acting

280 directly on the plasticity and not, in contrast, to a model of stabilizing selection

281 generated via pleiotropy [32].

282 We detected values of $\mathrm{V}_{\mathrm{g}} / \mathrm{V}_{\mathrm{m}}$ in areas of largest plasticity are substantially

283 lower than previously reported data (e.g., [45]). Thus, the question arises why

284 mutational variance among clonally related strains is larger than the observed

285 genetic variation among individuals from the outbred population in the area of

286 greatest plasticity. Our analyses indicate that neither experimental artifacts nor

287 maternal effects are the sole drivers of the observed substantial variation among

288 clonally related strains (Fig. $3 A$ ) and the small values of $V_{g} / V_{m}$ (Fig. 3B).

289 Consequently, we can only speculate on potential factors driving the observed

290 pattern. The outbred population is derived from individuals that recently hatched

291 from sexual ephippia deposited sometime in the past, whereas the clonally related

292 strains were present in the population an extended period of time leading up to the

293 point of collection. If the strength of predation varies through time, selection events

294 further in time in the past may have depleted diversity in clones that result from

295 sexual reproduction (i.e., our outbred population), while clonally strains may reflect

296 more recent population history. Although the exact mechanism of the large among-

297 line variance of the genetically similar clones is unclear, our analysis (Fig. 3)

298 suggests that it is not solely generated by experimental artifact or maternal effects. 
299 Examination of the evolutionary forces acting on plasticity is important for the

300 interpretation of the evolutionary history of this population and also for assessing

301 its evolutionary potential. While our data show that plasticity is subject to strong

302 stabilizing selection, we also show that there is ample mutational variance for

303 plasticity (Fig. 3). Mutational variance for plasticity could facilitate rapid adaptive

304 evolution following shifts in the predator composition in the aquatic community due

305 to climate change [48,49] or other anthropogenic factors (e.g., [50]), and could

306 thus be an important factor facilitating population persistence of $D$. pulex and other

307 organisms, with consequences for ecosystem stability and function [51].

310 Materials and Methods

\section{Study system}

312 Our data come from a population of Daphnia pulex located in the Kilwood Coppice

313 Nature Reserve in the Dorset region of the southern UK (grid reference: SY 93599

314 82555). Genotypes used in this study were sampled from two partly interconnected

315 seasonal ponds with predominantly invertebrate predators during early spring in

3162016 and 2017 (Suppl. Table 2). In the lab, sampled live individuals were

317 established as iso-female clonal lineages and maintained in artificial hard water

318 (ASTM; [52]) with seaweed extract (marinure; Wilfrid Smith Std., Northans, UK)

319 under standard conditions: 15 animals $\mathrm{L}^{-1}$ ASTM were fed three times a week with

320 Chlorella vulgaris $\left(2 \times 10^{5}\right.$ cells $\mathrm{ml}^{-1} ;>1.5 \mathrm{mg}$ carbon $\left.\mathrm{L}^{-1}\right)$ and reared under 16:8h

321 light:dark conditions at $20^{\circ} \mathrm{C}$. 


\section{Genotyping}

324 Sequencing: For DNA extractions, multiple adult Daphnia were placed into

325 artificial hard water containing antibiotics (Streptomycin, Tetracycline, and

326 Ampicillin, $50 \mathrm{mg} \mathrm{L}^{-1}$ of each) and fed Sephadex G-25 Superfine (cross-linked

327 dextran gel) beads for 48 hours in order to minimize bacterial and algal

328 contamination in downstream sequencing analyses. 5-10 individuals from each

329 clonal lineage were then used for DNA extraction using Beckman-Coulter's

330 Agencourt DNAdvance kit. Individuals were homogenized using metal beads and

331 a bead beater prior to DNA extraction. RNA was removed using RNase followed

332 by an additional bead clean-up. DNA was quantified using the broad-range Quant-

333 iT dsDNA kit (ThermoFisher Scientific) and an ABI plate reader and normalized to

3341 or $2 \mathrm{ng} \mathrm{ul}^{-1}$ prior to library construction. Full genome libraries were constructed

335 using a modified down Nextera protocol [53]. Libraries were size selected for

336 fragments ranging from 450-550bp using Blue Pippin and quality checked using

337 BioAnalyzer. Libraries were sequenced using the Illumina HiSeq 2500 platform.

338 Mapping, SNP calling, and SNP filtering: Nextera adaptor sequences were

339 removed using Trimmomatic version 0.36 [54] and overlapping reads were merged

340 using PEAR version 0.9.11 [55]. Assembled and unassembled reads were

341 separately mapped to a European $D$. pulex reference genome ([31]) using bwa

342 mem [56]. The entire reference genome was used for mapping, but only reads that

343 mapped to Daphnia scaffolds, had quality scores greater than 20, and were

344 primary alignments were used for further analysis. PCR duplicates were removed 
345 using Picard's MarkDuplicates function [57]. GATK HaplotypeCaller (version 4.0,

$346[58,59])$ was used to call SNPs. We removed SNPs that were within 10 base pairs

347 of indels. SNPs were then hard filtered using GATK's recommendations for

348 organisms with no reference SNP panel (QD $<2, \mathrm{FS}>60, \mathrm{MQ}<40, \mathrm{MQRankSum}$

$349<-12.5$, and ReadPosRankSum < -8). Individual genotype calls with low quality

350 scores $(\mathrm{GQ}<10)$ were set as missing data.

351 Clonal assignment: Individual field isolates were assigned to clonal lineages

352 based on patterns of identity by state (IBS). IBS was calculated using the

353 snpgdsIBS function in SNPRelate [60], with a minor allele frequency cutoff of 0.001

354 and a missing rate of 0.15 . We classified individual field isolates as coming from

355 the same clonal lineage if pairwise identity by states was greater than 0.965 (see

356 [31] for more details). We identified 50 genetically unique clonal groups, of which

357 one cluster was represented by 56 genetically similar strains, yielding 105 strains

358 that were used for phenotypic analysis. We investigated patterns of relatedness by

359 calculating IBSO and kinship coefficients using the program KING [61]. KING was

360 run using the "kinship" command with the input data filtered to include SNPs with

361 a minor allele frequency cutoff of 0.05 .

363 Phenotyping

364 Experimental exposures: Phenotypic data were collected from 105 iso-female

365 lines from the Killwood Nature Preserve (Suppl. Tab. 2). To establish predation

366 risk conditions, we generated predator cues from frozen midge larvae following

367 established protocols [62]. Homogenized midge larvae extracts were filtered, 
368 followed by solid-phase extraction using a $\mathrm{C}_{18}$ column (Agilent) to recover the

369 active compounds that generate strong morphological responses in D. pulex. For

370 experimental exposures, animals were kept under standard conditions for three

371 generations. Subsequently, at least two gravid Daphnia, carrying embryos in $\mathrm{E}_{3}$

372 stage ( 18 hours before parturition; sensu [63]), were placed in individual jars

373 containing $50 \mathrm{ml}$ hard artificial pond water [52], algae $\left(2 \times 10^{5}\right.$ cells $\mathrm{ml}^{-1}$ Chlorella

374 vulgaris), liquid seaweed extract and 0 or $0.5 \mu \mathrm{ml}^{-1}$ Chaoborus predator cue

375 concentrate. After parturition, two neonates were randomly selected from each of

376 the two mothers per treatment (Suppl. Fig. 2) and placed individually in $50 \mathrm{ml}$ glass

377 vials containing the same medium as their maternal environment. For three to four

378 consecutive days, each animal was photographed daily (Leica S8AP0 microscope;

379 Leica EC4 camera) and subsequently transferred to a new glass vial containing

380 fresh media and predator cues.

381 High-throughput image analysis: We assessed phenotypic changes using an

382 automated image analysis pipeline hereafter referred to as DAPCHA (see Suppl.

383 Methods). To validate accurate performance of $D A P C H A$, all images were

384 manually checked, and if required, landmarks manually curated. We recorded

385 manual estimates of units of microstage meter (>100 randomly chosen images)

386 via two separate runs of estimation, and animal lengths and morphological

387 induction (>700 randomly chosen images) via three independent observers using

388 ImageJ software ([64]; Fiji plugin: [65]). We manually measured animal length from

389 the tip of the head to the base of the tail. Morphological induction, based on the

390 presence of a pedestal, was manually scored using a previously defined scoring 
391 system [28, 29]. Developed spikes in the nuchal area, later referred to as

392 neckteeth, were counted individually.

394 Instar assignment: Animals were retroactively assigned to distinct developmental

395 stages (i.e., first and second instar) by fitting a mixture model on animal dorsal

396 area and animal length, using the Mclust package for $\mathrm{R}$ [66].

398 Analysis of variance: To assess the contribution of genotype, treatment, and their

399 interaction, we fit a linear model (dorsal height $\sim$ genotype * treatment + batch),

400 followed by Type II sums of squares implemented by the Anova() function in the

401 car package for $\mathrm{R}$ [67] for significance testing, and estimation of effect sizes using

402 the effectsize package for $\mathrm{R}$ [68].

404 Magnitude and position of induced defense: To estimate how predation risk 405 altered the magnitude and position of the morphological defense structures, we 406 applied a phenotypic trajectory analysis [69] to the multivariate data matrix of 407 dorsal height at each $i^{\text {th }}$ position along the dorsal edge using the geomorph 408 package for $\mathrm{R}$ [70-72]. We fit a model where the response variable is the 409 multivariate dorsal height $\mathrm{x}$ position matrix among genotypes versus treatment

410 (control - predation). We estimated the overall impact of predation risk using 1000

411 permutations via the procD. $\operatorname{Im}()$ function. This was followed by assessment of the 412 direction and magnitude statistics via the trajectory.analysis() function. 
413 Visualization of the morphology and details about the shift in height and position

414 of maximal induction were made with the plotRefToTarget() function.

415 To assess modularity and identify whether the region where the morphological

416 defense is induced is correlated (or not) with the rest of the body, we applied the

417 modularity.test() function in the geomorph package for R [70-72]. We tested for the

418 presence of modularity between areas of largest plasticity (i.e., head area) and the

419 remaining dorsal areas along the carapace.

421 Correlation analysis: To assessed how much offspring resembled one another

422 when released from (i) the same mother and the same clutch, (ii) the same strain

423 but different mother, (iii) randomly drawn strains from the clonal assemblage of

424 genetically related strains, and (iv) randomly drawn individuals from each

425 experimental batch, we performed a correlation analysis for two key phenotypic

426 traits, animal length and maximal dorsal height, using the $\mathrm{R}$ package robcor [73].

427 In order to estimate correlation coefficients for randomly drawn clone pairs from

428 among genetically similar strains or batches, we performed 100 bootstraps. We

429 also permuted phenotypic data in order to obtain a NULL distribution of correlation

430 coefficients $(n=1000)$.

432 Heritability estimation: We estimated broad-sense heritability of phenotypic traits

433 using the R package MCMCglmm [74]: dorsal height 1, clone + batch. Models

434 were fitted with a burn-in of 15,000 and sampling that produced 1300 estimates of

435 the posterior distribution from 65,000 iterations of the chain. All models were 
436 checked for autocorrelation in the chains. We calculated $V_{g} / V_{m}$ as

437 mean( $\log _{10}$ (posterior distribution standing variation) - $\log _{10}($ posterior distribution mutational

438 variation)).

439 We next estimated narrow-sense heritability of phenotypic traits in the 440 genetically divergent clones using GCTA [75]. We generated a set of genome-wide

441 representative SNPs, and then calculated a genetic relatedness matrix from these

442 SNPs with the --make-grm flag in GCTA. For heritability estimations, we used the

443 flags --reml, --reml-alg 1, accounting for batch as covariate. In order to estimate

444 heritability estimates for random data (i.e., NULL distribution), we permuted

445 genome identifiers before calculating genetic relatedness matrices.

447 Statistical analysis and plotting: All analyses were performed using R version

4483.5 [76]. The following packages were used for general analysis and plotting:

449 ggplot2 [77], cowplot [78], data.table [79], foreach [80], doMC [81], ggbeeswarm

$450 \quad$ [82], and viridis [83].

451

452 Data Availability: All scripts and code used for data analysis and plotting are 453 available at https://github.com/beckerdoerthe/SelectionPlasticity. DAPCHA is 454 available at https://github.com/beckerdoerthe/Dapcha_v.1. All raw images and 455 processed data used to generate figures are deposited in Zenodo (DOI $45610.5281 /$ zenodo.4738526). All sequencing reads are available from the Sequence 457 Read Archive (PRJNA725506). 
459 Acknowledgments: AOB was supported by the National Institutes of Health (R35

460 GM119686) and by start-up funds provided by the University of Virginia. This

461 project has received funding from the European Union's Horizon 2020 research

462 and innovation programme under the Marie Sklodowska-Curie grant agreement

463 No 841419. The authors acknowledge Research Computing at The University of

464 Virginia for providing computational resources and technical support that have

465 contributed to the results reported within this publication (https://rc.virginia.edu).

466 The authors would also like to thank the Dorset Wildlife Trust for granting access

467 to the field site.

469 Author Contributions: DB: Conceptualization, Data curation, Formal analysis,

470 Investigation, Methodology, Resources, Software, Visualization, Writing - original

471 draft, Writing - review \& editing; KBK: Investigation, Resources, Writing - review \&

472 editing; RP: Investigation, Writing - review \& editing; $\underline{\mathrm{AE}}$ : Investigation, Writing -

473 review \& editing; EV: Investigation, Writing - review \& editing; $\underline{\text { APB: }}$

474 Conceptualization, Resources, Writing - review \& editing; $\underline{\text { AOB: }}$ : Conceptualization,

475 Data curation, Formal analysis, Funding acquisition, Investigation, Methodology,

476 Project administration, Resources, Software, Supervision, Visualization, Writing -

477 original draft, Writing - review \& editing.

479 Competing Interest Statement: All authors declare no competing interests. 


\section{Figures and Tables}

A

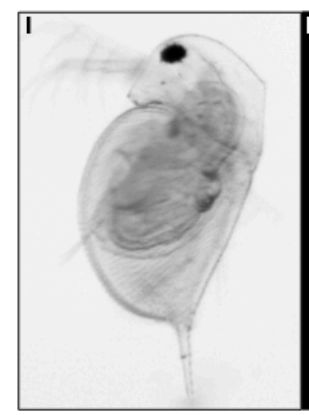

B

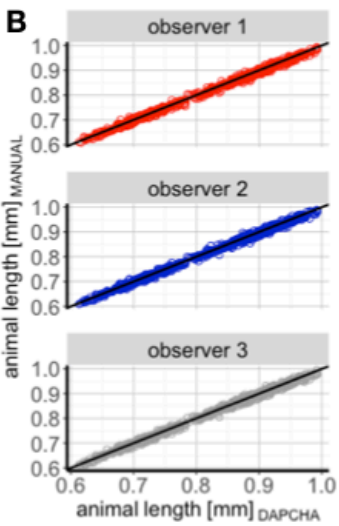

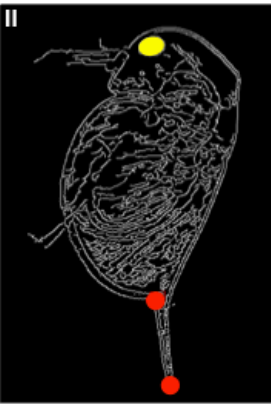

III
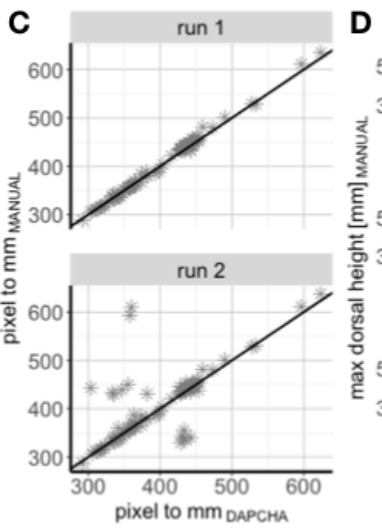
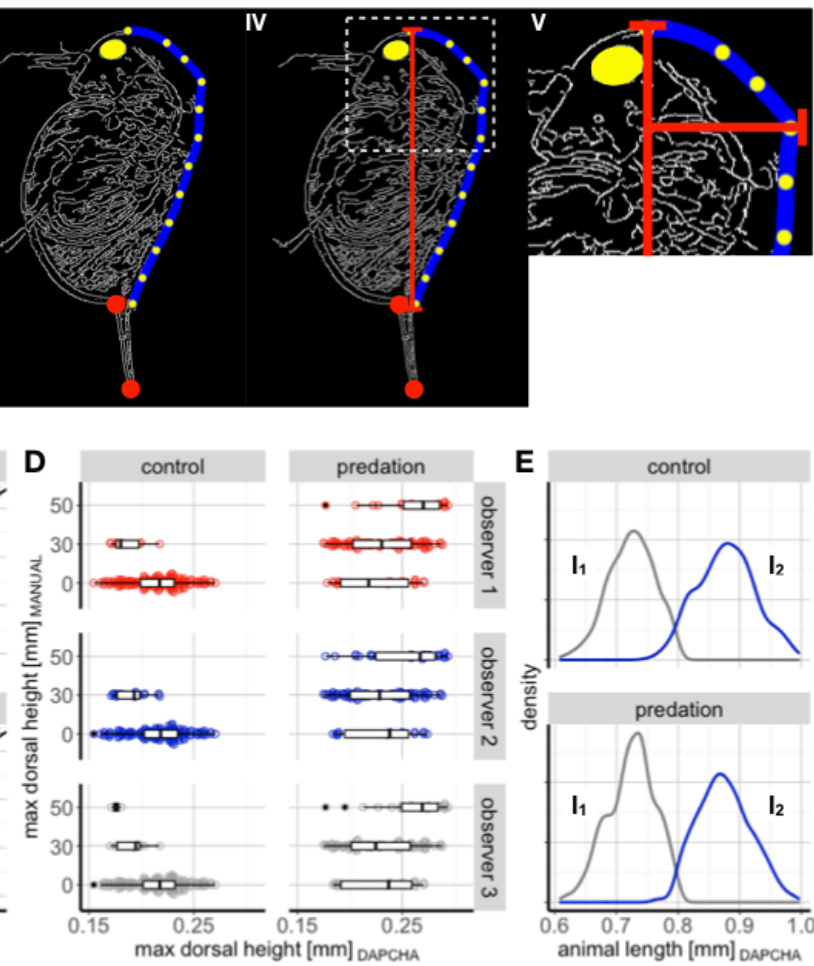

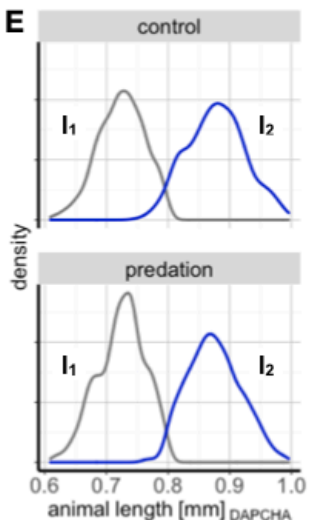

Figure 1. High-throughput phenotypic assessment via novel, automated image analysis tool 'DAPCHA'. (A) Phenotypic assessment of Daphnia using DAPCHA involves three major steps: conversion of a standardized, raw image to greyscale (I-II); automated identification of key landmarks (i.e., eye, tail tip and tail base) (II); and automated tracing of the dorsal edge of the carapace (blue line) via identification of equally spaced landmarks along the dorsal axis (yellow points) (III). Defined landmarks subsequently allow for the quantification of different phenotypic traits, including animal length (IV) and dorsal height ( $\mathrm{V}$; here exemplified by the dorsal position where dorsal height was largest). (B-D) Accuracy of phenotypic estimates by DAPCHA were validated via contrasting manual estimates with automated data: animal length estimates across three different observers (B), unit conversion of length estimates using a microstage meter assessed in two different runs (see Materials and Methods) (C), and morphological changes in the nuchal area under control and predation conditions estimated by three different observers (D). (E) Using a mixture model on animal length and animal area, test animals were retroactively assigned to distinct developmental stages (i.e., first instar, $\mathrm{I}_{1}$; second instar, $\mathrm{I}_{2}$ ). 

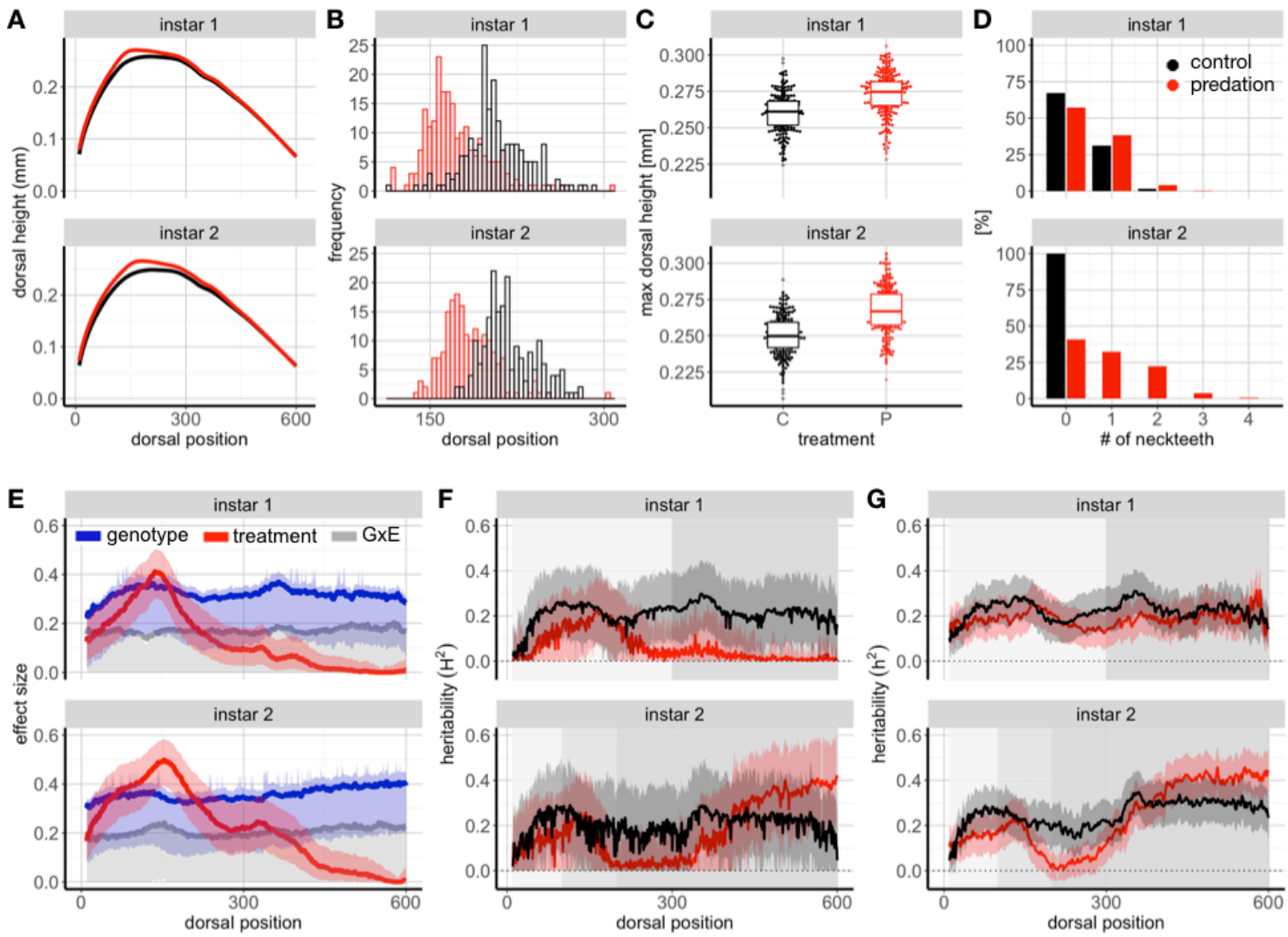

Figure 2. Effects of predation risk on morphological changes in genetically unique strains.

(A) Risk of predation induces marked shape changes along the dorsal axis in first and second instar D. pulex (control: black line; predation: red line). (B) Strongest morphological changes are observed in the head area, with maximal dorsal height shifting towards anterior head regions under predation risk. (C) Predator induced defense morphologies, here measured as maximal dorsal height, 504 increase in response to predation risk exposure in both instars (control, C: black points; predation, P: red points). (D) The number of neckteeth increases in response to predation risk exposure, particularly in second instar animals. (E) Effect sizes from a linear model along the dorsal axis reveal distinct patterns of treatment (i.e., predation risk, red line), genotype (blue line), and GxE (grey line) effects on morphological changes. Shaded areas indicate upper (0.95) and lower (0.05) confidence intervals. $(F, G)$ Broad sense $(F)$ and narrow sense $(G)$ heritability estimates of dorsal height vary along the dorsal axis in response to control conditions (black line) and predation risk (red line), with a strong reduction of both measures of heritability for dorsal height upon predation risk exposure in the region of maximum plasticity (i.e., dorsal positions 100-250). Shaded areas indicate upper (0.95) and lower (0.05) confidence intervals $(F)$ or standard errors $(G)$. Grey rectangles in panels $\mathrm{F}$ and $\mathrm{G}$ highlight morphological independent shape modules, separating head and posterior body areas (see Suppl. Fig. 4). 
A

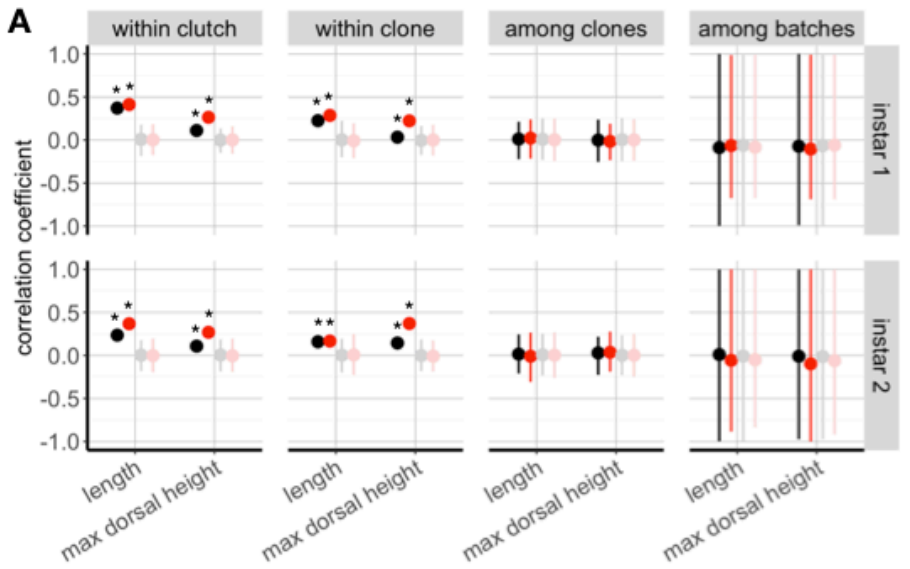

B

B instar

instar 1

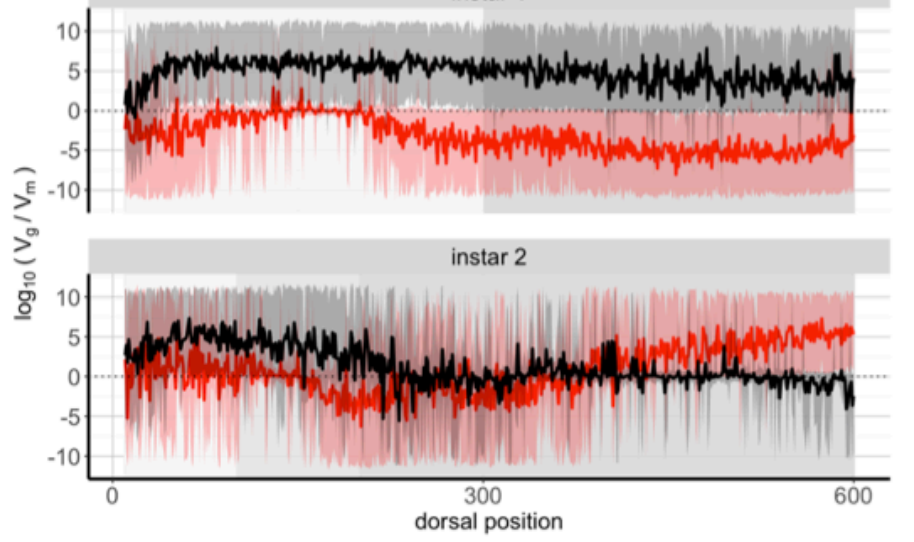

517 Figure 3. Effects of natural selection on predator induced plasticity in Daphnia. (A)

518 Phenotypic variation in genetically similar strains arises due to genetic effects: phenotypic responses (here: animal length and maximal dorsal height) of offspring released from the same mother ('within clutch') and same strain ('within clone') are more similar to each other than to offspring released from a randomly drawn member of the clonal assemblage ('among clones'). Correlation coefficients broadly exceed coefficients calculated for permuted data. Moreover, phenotypic correlations among randomly paired individuals from the same experimental batches are low, with actual data not exceeding permuted data ranges. Black and red points indicate control and predation risk conditions with darker and lighter colors depicting actual and permuted data, respectively. Error bars indicate upper (0.95) and lower (0.05) confidence intervals. Asterisks indicate actual data exceeding permuted data ranges (see Suppl. Tab. 1). (B) $\log _{10}\left(V_{g} / V_{m}\right)$ estimates of dorsal height vary along the dorsal axis in response to control conditions (black line) and predation risk (red line). Notably, in second instar Daphnia, genetic diversity is strongly reduced in areas of largest phenotypic plasticity. Shaded areas indicate upper (0.95) and lower (0.05) confidence intervals. Grey rectangles highlight morphological independent shape modules, separating head and posterior body areas (see Suppl. Fig. 4). 


\section{Supplemental Information}

536 Supplemental Table 1. List of statistical tests outcomes.

\section{DAPCHA validation (Fig. 1)}

(1.1): animal length observer 1 vs DAPCHA observer 2 vs DAPCHA observer 3 vs DAPCHA

(1.2): microstage meter observer vs DAPCHA, run 1 observer vs DAPCHA, run 2

(1.3): defense morphologies max dorsal height $\sim$ treatment

max dorsal height MANUAL vs max dorsal heightDAPCHA max dorsal heightmANUAL $v s$ max dorsal heightDAPCHA, 2nd instar only

two-way intraclass correlation, type='agreement'

two-way intraclass correlation, type='agreement', control only

two-way intraclass correlation, type='agreement', predation only

\section{Morphological induction (Fig. 2)}

(2.1): dorsal height shift instar 1 instar 2

(2.2): dorsal height increase instar 1 instar 2

\section{(2.3): \# neckteeth increase} instar 1 instar 2

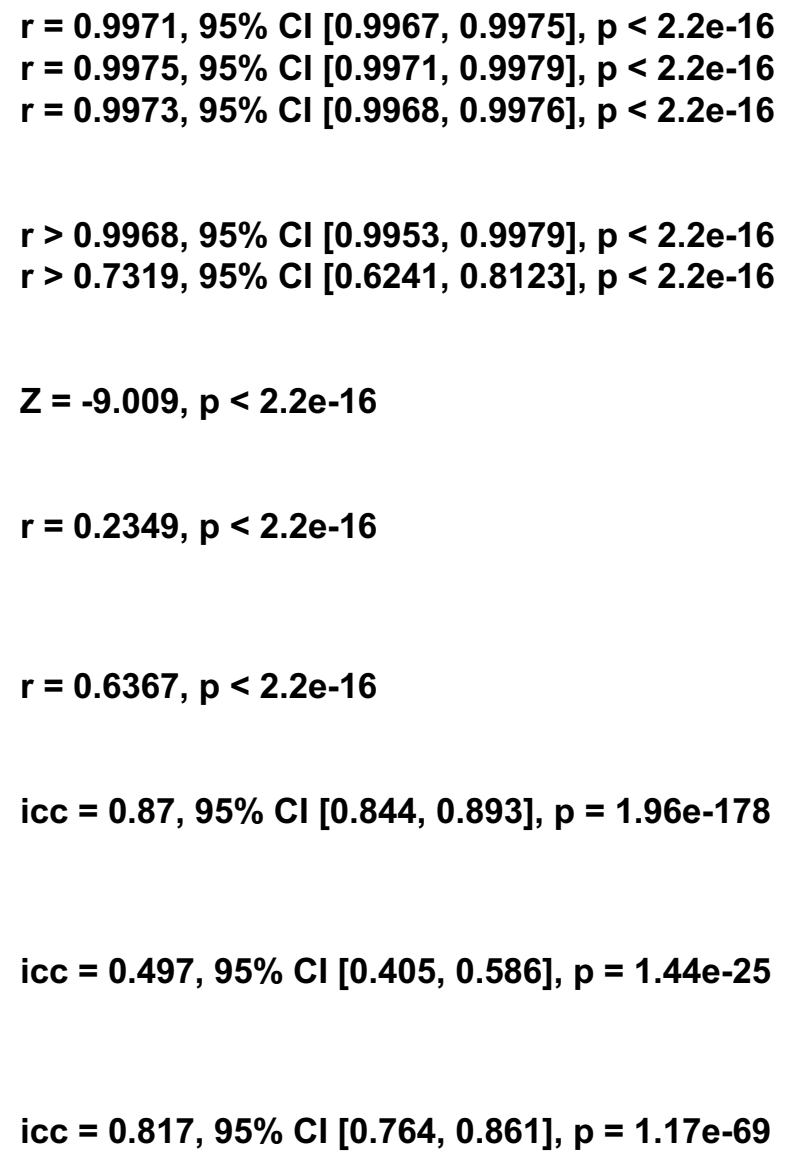

$\chi^{2}[\mathrm{df}=121, \mathrm{~N}=411]=210.3, \mathrm{p}=\mathbf{8 . 9 2 6 e - 0 7}$

$\chi^{2}[\mathrm{df}=112, \mathrm{~N}=380]=222.4, \mathrm{p}=2.676 \mathrm{e}-09$

$Z=-8.959, p<2.2 e-16$

$Z=-9.836, p<2.2 e-16$

$\chi^{2}[\mathrm{df}=6, \mathrm{~N}=412]=7.84, \mathrm{p}=0.25$

$\chi^{2}[\mathrm{df}=7, \mathrm{~N}=381]=179.83, \mathrm{p}<2.2 \mathrm{e}-16$ 
(2.4): heritability estimates decrease

$\mathrm{H}^{2}$, instar 1

$\mathrm{H}^{2}$, instar 2

$\mathrm{h}^{2}$, instar 1

$\mathrm{h}^{2}$, instar 2

\section{Twin analysis (Fig. 3)}

(3.1): actual vs permuted data (3.1.1) within clutch - animal length instar 1 , control instar 1 , predation instar 2, control instar 2, predation

(3.1.2) within clutch - max dorsal height instar 1 , control instar 1, predation instar 2, control instar 2, predation

\section{(3.1.3) within clone - animal length}

instar 1 , control instar 1 , predation instar 2, control instar 2, predation

\section{(3.1.4) within clone - max dorsal height} instar 1 , control instar 1 , predation instar 2, control instar 2, predation

\section{(3.1.5) among clones - animal length}

instar 1 , control instar 1, predation

instar 2, control instar 2, predation

(3.1.6) among clones - max dorsal height instar 1 , control instar 1 , predation instar 2, control instar 2, predation

\section{(3.1.7) among batches - animal length}

instar 1 , control instar 1 , predation

instar 2, control

instar 2, predation

$$
\begin{aligned}
& Z=-10.996, p<2.2 e-16 \\
& Z=-7.129, p=1.013 e-12 \\
& Z=-5.071, p=3.949 e-07 \\
& Z=-10.405, p<2.2 e-16
\end{aligned}
$$

$Z=-27.39292, p<2.2 e-16$
$Z=-27.39292, p<2.2 e-16$
$Z=-27.32505, p<2.2 e-16$
$Z=-27.39292, p<2.2 e-16$

$Z=-25.78852, p<2.2 e-16$

$Z=-27.39292, p<2.2 e-16$

$Z=-21.86441, p<2.2 e-16$

$Z=-27.35843, p<2.2 e-16$

$Z=-26.87691, p<2.2 e-16$

$Z=-27.38482, p<2.2 e-16$

$Z=-25.83406, p<2.2 e-16$

$Z=-23.86439, p<2.2 e-16$

$Z=-10.8457, p<2.2 e-16$
$Z=-27.35712, p<2.2 e-16$
$Z=-25.66034, p<2.2 e-16$
$Z=-27.39292, p<2.2 e-16$

$Z=-0.1025878, p=0.9183$

$Z=-0.7975722, p=0.4251$

$Z=-1.06435, p=0.2872$

$Z=-0.8084267, p=0.4188$

$Z=-0.1169427, p=0.9069$

$Z=-1.161296, p=0.2455$

$Z=-0.4066517, p=0.6843$

$Z=-1.811072, p=0.07013$

$Z=-0.422821, p=0.6724$

$Z=-1.350417, p=0.1769$

$Z=-1.004316, p=0.3152$

$Z=-0.6890701, p=0.4908$ 
(3.1.8) among batches - max dorsal height

instar 1 , control

instar 1 , predation

instar 2, control

instar 2, predation

$$
\begin{aligned}
& Z=-0.6746085, p=0.4999 \\
& Z=-1.497639, p=0.1342 \\
& Z=-0.1886146, p=0.8504 \\
& Z=-\mathbf{2 . 8 0 6 0 0 3 , p = 0 . 0 0 5 0 1 6}
\end{aligned}
$$

(3.2): within clutch vs among clones

\section{(3.2.1) animal length}

instar 1 , control

instar 1, predation

instar 2, control

instar 2, predation

(3.2.2) max dorsal height

instar 1 , control

instar 1 , predation

instar 2, control

instar 2, predation

(3.3): within clone vs among clones

(3.3.1) animal length

instar 1, control

instar 1 , predation

instar 2, control

instar 2, predation

\section{(3.3.2) max dorsal height}

instar 1 , control

instar 1 , predation

instar 2, control

instar 2, predation
$Z=-8.680051, p<2.2 e-16$

$Z=-8.676613, p<2.2 e-16$

$Z=-8.37404, p<2.2 e-16$

$Z=-8.676613, p<2.2 e-16$

$Z=-5.984404, p=2.172 \mathrm{e}-09$
$Z=-8.676613, p<2.2 \mathrm{e}-16$
$Z=-5.929391, p=3.041 \mathrm{e}-09$
$Z=-8.494382, p<2.2 \mathrm{e}-16$

$Z=-8.542518, p<2.2 e-16$

$Z=-8.583778, p<2.2 e-16$

$Z=-7.033093, p=2.02 e-12$

$Z=-7.531651, p=5.01 e-14$

$Z=-1.720882, p=0.08527$

$Z=-8.631915, p<2.2 e-16$

$Z=-7.294406, p=3 e-13$

$Z=-8.676613, p<2.2 e-16$

\section{Morphological induction (Suppl. Fig. 5)}

(S5.1): dorsal height shift

instar 1

instar 2

$\chi^{2}[\mathrm{df}=119, \mathrm{~N}=463]=284.84, \mathrm{p}=1.267 \mathrm{e}-15$

$\chi^{2}[\mathrm{df}=106, \mathrm{~N}=433]=228.35, \mathrm{p}=5.758 \mathrm{e}-11$

(S5.2): dorsal height increase

instar 1

$Z=-8.722, p<2.2 e-16$

instar 2

$Z=-10.455, p<2.2 e-16$

(S5.3): \# neckteeth increase

instar 1

$\chi^{2}[\mathrm{df}=4, \mathrm{~N}=464]=10.588, \mathrm{p}=0.0316$

instar 2

$\chi^{2}[\mathrm{df}=7, \mathrm{~N}=431]=242.64, \mathrm{p}<2.2 \mathrm{e}-16$ 
538 Supplemental Table 2. List of Daphnia pulex clones used in this study, including information

539 on pond identity and sampling season. Cluster information indicates whether strains are genetically

540 similar ('cluster A') or genetically unique ('cluster O').

\section{Clone ID}

April_2017_D8_101

April_2017_D8_103

April_2017_D8_119

April_2017_D8_134

April_2017_D8_135

April_2017_D8_136

April_2017_D8_137

April_2017_D8_141

April_2017_D8_142

April_2017_D8_147

April_2017_D8_151

April_2017_D8_157

April_2017_D8_175

April_2017_D8_179

April_2017_D8_183

April_2017_D8_191

April_2017_D8_201

April_2017_D8_202

April_2017_D8_203

April_2017_D8_209

April_2017_D8_211

April_2017_D8_213

April_2017_D8_215

April_2017_D8_223

April_2017_D8_227

April_2017_D8_248

April_2017_D8_254

April_2017_D8_256

April_2017_D8_298

April_2017_D8_338

April_2017_D8_342

April_2017_D8_349

April_2017_D8_360

April_2017_D8_399

April_2017_D8_60

April_2017_D8_77

April_2017_DBunk_112

April_2017_DBunk_119

April_2017_DBunk_13

April_2017_DBunk_131

April_2017_DBunk_132

April_2017_DBunk_160

April_2017_DBunk_248

April_2017_DBunk_254

April_2017_DBunk_26

April_2017_DBunk_297

\begin{tabular}{|c|c|c|}
\hline $\begin{array}{l}\text { Pond } \\
\text { D8 }\end{array}$ & $\begin{array}{l}\text { Cluster } \\
\mathrm{O}\end{array}$ & $\begin{array}{l}\text { Season } \\
\text { Spring } 2017\end{array}$ \\
\hline D8 & A & Spring 2017 \\
\hline D8 & $A$ & Spring 2017 \\
\hline D8 & 0 & Spring 2017 \\
\hline D8 & $A$ & Spring 2017 \\
\hline D8 & $A$ & Spring 2017 \\
\hline D8 & 0 & Spring 2017 \\
\hline D8 & $A$ & Spring 2017 \\
\hline D8 & A & Spring 2017 \\
\hline D8 & $A$ & Spring 2017 \\
\hline D8 & $\mathrm{O}$ & Spring 2017 \\
\hline D8 & A & Spring 2017 \\
\hline D8 & A & Spring 2017 \\
\hline D8 & A & Spring 2017 \\
\hline D8 & A & Spring 2017 \\
\hline D8 & A & Spring 2017 \\
\hline D8 & A & Spring 2017 \\
\hline D8 & A & Spring 2017 \\
\hline D8 & A & Spring 2017 \\
\hline D8 & A & Spring 2017 \\
\hline D8 & A & Spring 2017 \\
\hline D8 & A & Spring 2017 \\
\hline D8 & A & Spring 2017 \\
\hline D8 & A & Spring 2017 \\
\hline D8 & A & Spring 2017 \\
\hline D8 & A & Spring 2017 \\
\hline D8 & $A$ & Spring 2017 \\
\hline D8 & A & Spring 2017 \\
\hline D8 & A & Spring 2017 \\
\hline D8 & $A$ & Spring 2017 \\
\hline D8 & A & Spring 2017 \\
\hline D8 & $A$ & Spring 2017 \\
\hline D8 & $A$ & Spring 2017 \\
\hline D8 & $A$ & Spring 2017 \\
\hline D8 & $A$ & Spring 2017 \\
\hline D8 & $A$ & Spring 2017 \\
\hline DBunk & 0 & Spring 2017 \\
\hline DBunk & 0 & Spring 2017 \\
\hline DBunk & $\mathrm{O}$ & Spring 2017 \\
\hline DBunk & $\mathrm{O}$ & Spring 2017 \\
\hline DBunk & $\mathrm{O}$ & Spring 2017 \\
\hline DBunk & $\mathrm{O}$ & Spring 2017 \\
\hline DBunk & $\mathrm{O}$ & Spring 2017 \\
\hline DBunk & $\mathrm{O}$ & Spring 2017 \\
\hline DBunk & $A$ & Spring 2017 \\
\hline DBunk & 0 & Spring 2017 \\
\hline
\end{tabular}




\begin{tabular}{|c|c|c|c|}
\hline April_2017_DBunk_302 & DBunk & $\mathrm{O}$ & Spring 2017 \\
\hline April_2017_DBunk_36 & DBunk & $\mathrm{O}$ & Spring 2017 \\
\hline April_2017_DBunk_6 & DBunk & $\mathrm{O}$ & Spring 2017 \\
\hline April_2017_DBunk_63 & DBunk & $\mathrm{O}$ & Spring 2017 \\
\hline April_2017_DBunk_9 & DBunk & 0 & Spring 2017 \\
\hline April_2017_DBunk_90 & DBunk & $\mathrm{O}$ & Spring 2017 \\
\hline April_2017_DBunk_91 & DBunk & $\mathrm{O}$ & Spring 2017 \\
\hline May_2017_D8_520 & D8 & A & Spring 2017 \\
\hline May_2017_D8_521 & D8 & A & Spring 2017 \\
\hline May_2017_D8_532 & D8 & A & Spring 2017 \\
\hline May_2017_D8_538 & D8 & 0 & Spring 2017 \\
\hline May_2017_D8_539 & D8 & A & Spring 2017 \\
\hline May_2017_D8_542 & D8 & A & Spring 2017 \\
\hline May_2017_D8_544 & D8 & 0 & Spring 2017 \\
\hline May_2017_D8_612 & D8 & A & Spring 2017 \\
\hline May_2017_D8_663 & D8 & A & Spring 2017 \\
\hline May_2017_D8_668 & D8 & A & Spring 2017 \\
\hline May_2017_D8_673 & D8 & A & Spring 2017 \\
\hline May_2017_D8_731 & D8 & $\mathrm{O}$ & Spring 2017 \\
\hline May_2017_D8_756 & D8 & A & Spring 2017 \\
\hline May_2017_D8_771 & D8 & A & Spring 2017 \\
\hline May_2017_D8_776 & D8 & A & Spring 2017 \\
\hline May_2017_DBūnk_514 & DBunk & $\mathrm{O}$ & Spring 2017 \\
\hline May_2017_DBunk_523 & DBunk & O & Spring 2017 \\
\hline May_2017_DBunk_525 & DBunk & $\mathrm{O}$ & Spring 2017 \\
\hline May_2017_DBunk_547 & DBunk & $\mathrm{O}$ & Spring 2017 \\
\hline May_2017_DBunk_558 & DBunk & $\mathrm{O}$ & Spring 2017 \\
\hline May_2017_DBunk_579 & DBunk & O & Spring 2017 \\
\hline May_2017_DBunk_590 & DBunk & $\mathrm{O}$ & Spring 2017 \\
\hline May_2017_DBunk_591 & DBunk & $\mathrm{O}$ & Spring 2017 \\
\hline May_2017_DBunk_594 & DBunk & A & Spring 2017 \\
\hline Spring_2016_D8_8.29 & D8 & $\mathrm{O}$ & Spring 2016 \\
\hline Spring_2016_D8_8.31 & D8 & $\mathrm{O}$ & Spring 2016 \\
\hline Spring_2017_D8_225 & D8 & A & Spring 2017 \\
\hline Spring_2017_D8_262 & D8 & A & Spring 2017 \\
\hline Spring_2017_D8_282 & D8 & A & Spring 2017 \\
\hline Spring_2017_D8_294 & D8 & A & Spring 2017 \\
\hline Spring_2017_D8_327 & D8 & A & Spring 2017 \\
\hline Spring_2017_D8_329 & D8 & A & Spring 2017 \\
\hline Spring_2017_D8_336 & D8 & $\mathrm{O}$ & Spring 2017 \\
\hline Spring_2017_D8_339 & D8 & A & Spring 2017 \\
\hline Spring_2017_D8_343 & D8 & A & Spring 2017 \\
\hline Spring_2017_D8_350 & D8 & A & Spring 2017 \\
\hline Spring_2017_D8_366 & D8 & A & Spring 2017 \\
\hline Spring_2017_DBunk_122 & DBunk & $\mathrm{O}$ & Spring 2017 \\
\hline Spring_2017_DBunk_143 & DBunk & $\mathrm{O}$ & Spring 2017 \\
\hline Spring_2017_DBunk_217 & DBunk & $\mathrm{O}$ & Spring 2017 \\
\hline Spring_2017_DBunk_242 & DBunk & $\mathrm{O}$ & Spring 2017 \\
\hline Spring_2017_DBunk_252 & DBunk & $\mathrm{O}$ & Spring 2017 \\
\hline Spring_2017_DBunk_260 & DBunk & $\mathrm{O}$ & Spring 2017 \\
\hline Spring_2017_DBunk_321 & DBunk & $\mathrm{O}$ & Spring 2017 \\
\hline Spring_2017_DBunk_338 & DBunk & $\mathrm{O}$ & Spring 2017 \\
\hline Spring_2017_DBunk_347 & DBunk & $\mathrm{O}$ & Spring 2017 \\
\hline Spring_2017_DBunk_360 & DBunk & $\mathrm{O}$ & Spring 2017 \\
\hline
\end{tabular}


bioRxiv preprint doi: https://doi.org/10.1101/2021.07.29.454146; this version posted July 29, 2021. The copyright holder for this preprint (which was not certified by peer review) is the author/funder. All rights reserved. No reuse allowed without permission.

Spring_2017_DBunk_363

Spring_2017_DBunk_367

Spring_2017_DBunk_378

Spring_2017_DBunk_387

Spring_2017_DBunk_73

$\begin{array}{lll}\text { DBunk } & \text { O } & \text { Spring 2017 } \\ \text { DBunk } & \text { O } & \text { Spring 2017 } \\ \text { DBunk } & \text { O } & \text { Spring 2017 } \\ \text { DBunk } & \text { O } & \text { Spring 2017 } \\ \text { DBunk } & \text { O } & \text { Spring 2017 }\end{array}$

542 


\section{Supplemental Figures}

544

545

A

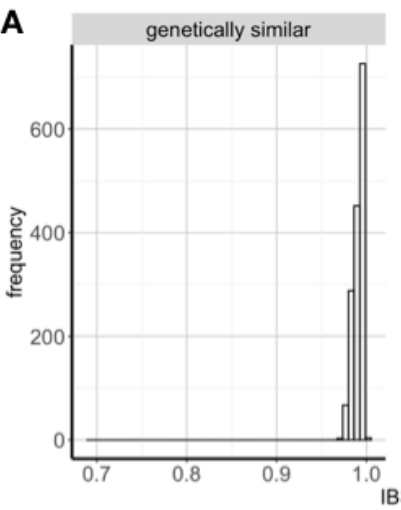

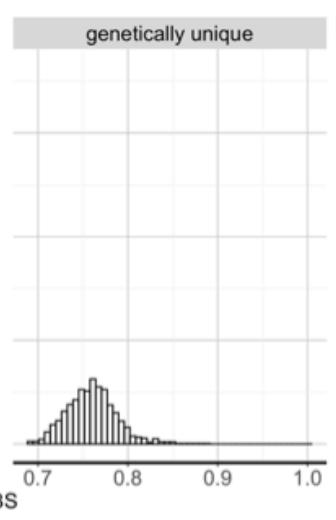

B

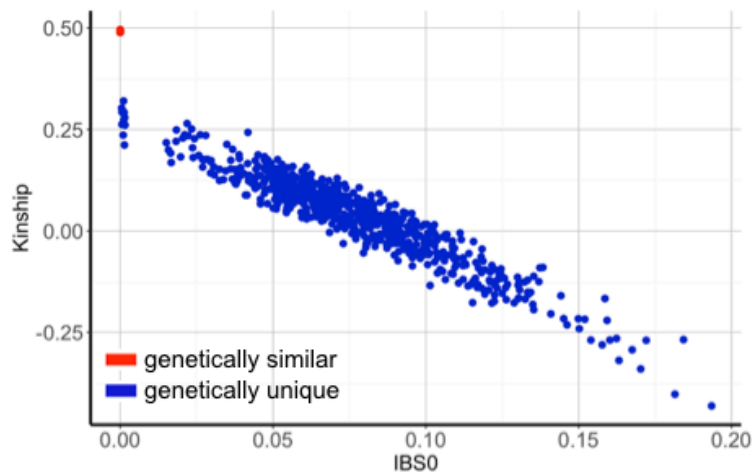

547 Supplemental Figure 1. Genetic diversity among genetically similar and unique strains. (A)

548 Distribution of pairwise IBS values between all genetically similar (left) and genetically unique (right)

549 strains. (B) Relationship between IBS0 and kinship as calculated in the program King for pairwise

550 combinations of individuals genotyped from the sampled population. Red and blue circles depict 551 genetically similar and unique strains, respectively. Note that in (B) all comparisons between 552 genetically similar strains are stacked on top of each other. 

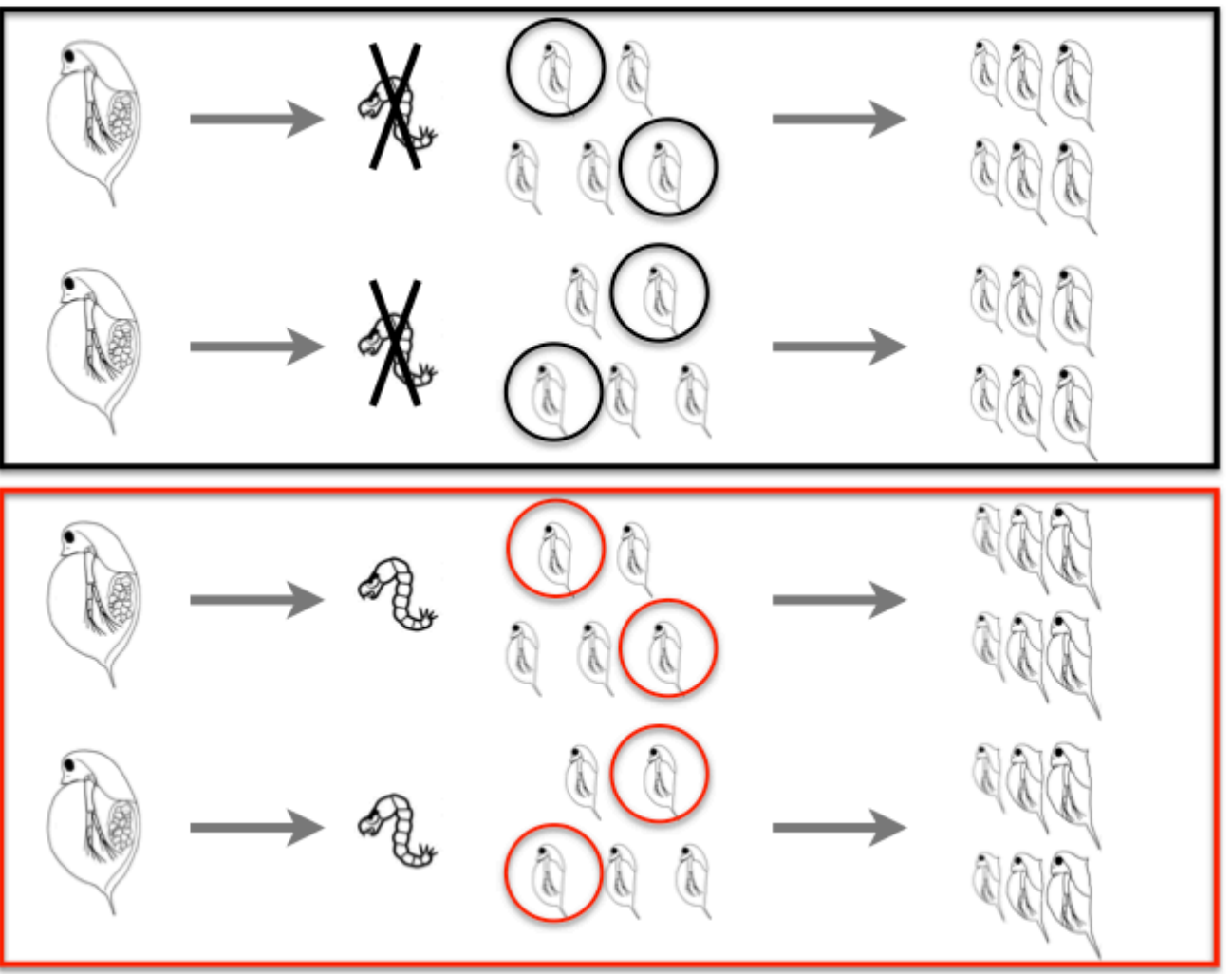

Supplemental Figure 2. Experimental design. For experimental exposures, two mature Daphnia pulex carrying embryos in E3 stage ( 18 hours before parturition; sensu [63]) were placed in individual jars containing medium with (red box) and without (black box) predator cues. After parturition, two neonates were randomly selected from each of the two mothers and placed in individual vials containing the same medium as their maternal environment. Subsequently, animals were monitored for 3-4 consecutive days, with daily photographs taken. 

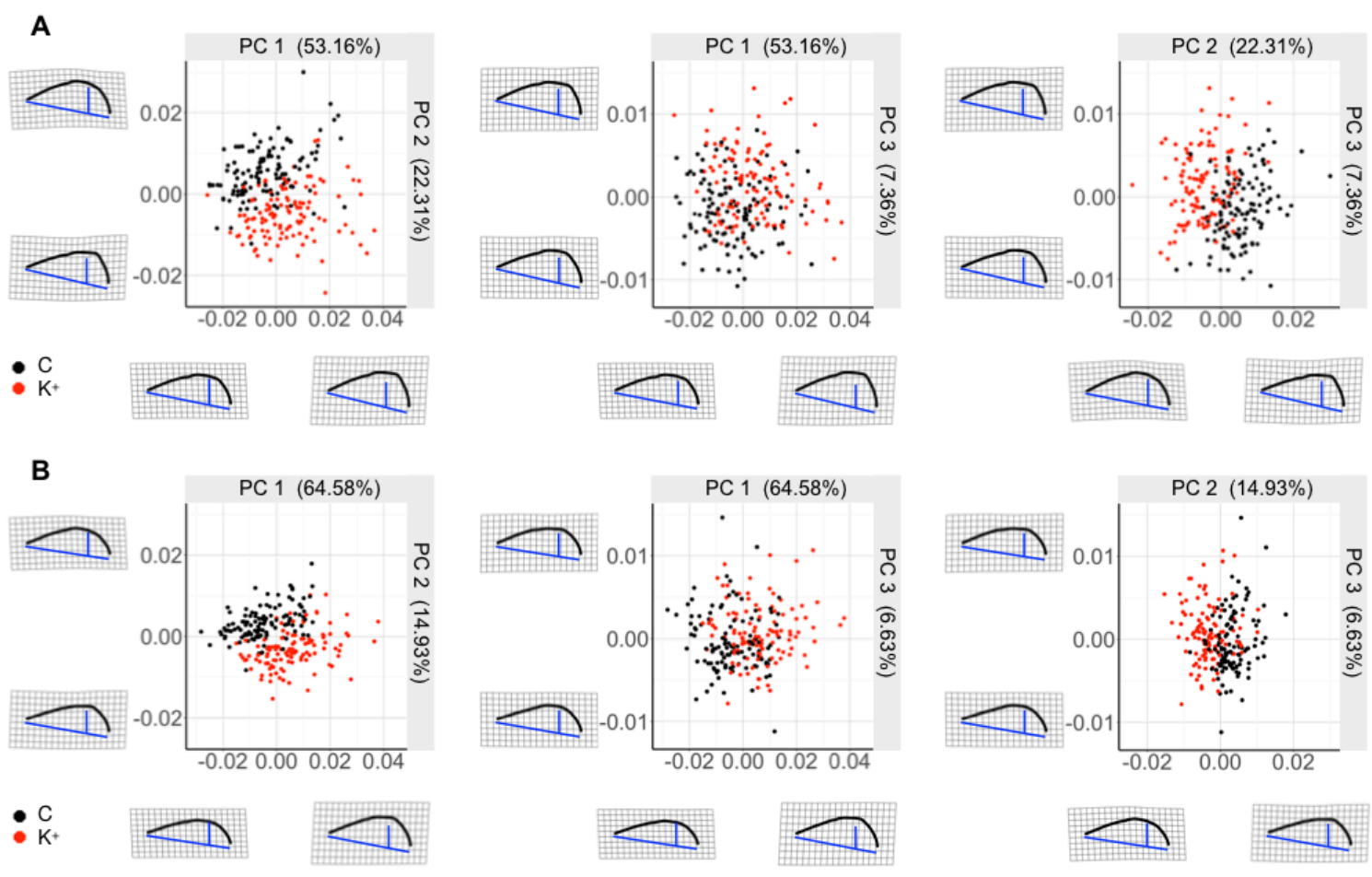

$:_{\mathrm{K}^{+}}^{\mathrm{c}}$
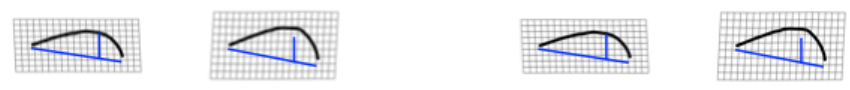

561 Supplemental Figure 3. Chaoborus induced shape variation in $\boldsymbol{D}$. pulex. Visualization of the first three main axes of dorsal shape variation in first (A) and second instar (B) Daphnia using principal component (PC) analysis of procrustes data. Colors indicate treatment conditions (control: black points, predation: red points). Warp-shape diagrams highlight distinctive patterns of shape variations along the principal components: PC1 represents shape differences in dorsal height, while PC2 and PC3 characterize the development of predator induced defense morphologies and shifts in their dorsal position, respectively. 
A

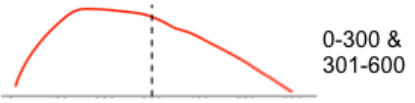

B

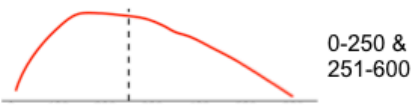

C

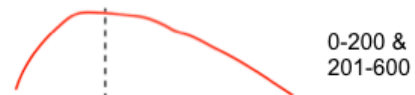

D

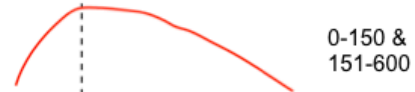

E

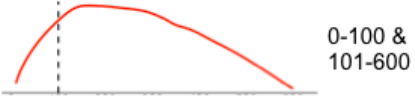

F

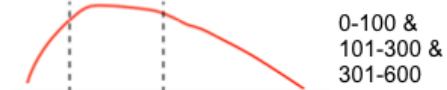

G

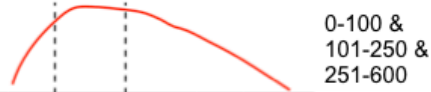

H

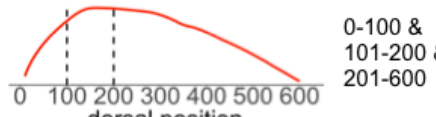

O cluster - instar 1 CR: 0.7061 p value: 0.001 effect size: -30.9874

CR: 0.726

p value: 0.001

effect size: -30.9269

CR: 0.7917

p value: 0.001

effect size: -30.2383

CR: 0.9148

p value: 0.001

effect size: -24.3989

CR: 0.914

p value: 0.001

effect size: - 21.3459

CR: 0.9267

p value: 0.001

effect size: -21.0665

CR: 0.7619

p value: 0.001

effect size: -30.032

CR: 0.7563

P value: 0.001

effect size: -30.1232

CR: 0.7619

p value: 0.001

effect size: -29.3356

effect size: -30.4054
CR: 0.8348

p value: 0.001

CR: 0.8297

p value: 0.001

effect size: -30.4785

CR: 0.8577

p value: 0.001

effect size: -29.5817

CR: 0.9174

p value: 0.001
effect size: -25.6406
CR: 0.8352

p value: 0.001

effect size: -29.3092

CR: 0.7938

p value: 0.001

effect size: -30.0756

CR: 0.7434

p value: 0.001

effect size: -29.9931
A cluster - instar 1 A cluster - instar 2 CR: $0.7622 \quad$ CR: 0.8392 p value: $0.001 \quad$ p value: 0.001 effect size: -30.991 effect size: -31.015

CR: $0.7872 \quad$ CR: 0.8477 p value: $0.001 \quad$ p value: 0.001

effect size: -30.9244 effect size: -30.9979

$\begin{array}{ll}\text { CR: } 0.837 & \text { CR: } 0.8848\end{array}$

p value: $0.001 \quad$ p value: 0.001

effect size: -30.2472 effect size: -30.3305

CR: $0.9048 \quad$ CR: 0.9562

p value: $0.001 \quad$ p value: 0.001

effect size: -27.1161 effect size: -23.9484

\begin{tabular}{l|l} 
CR: 0.9191 & CR: 0.9595
\end{tabular}

p value: $0.001 \quad$ p value: 0.001

effect size: -22.2475 effect size: -19.3365

CR: $0.7986 \quad$ CR: 0.8702

p value: $0.001 \quad$ p value: 0.001

effect size: -30.2594 effect size: -30.0785

CR: $0.7691 \quad$ CR:0.8466

p value: $0.001 \quad$ p value: 0.001

effect size: -30.356 effect size: -30.4998

Supplemental Figure 4. Modularity of predator induced defenses along dorsal axis. A formal modularity analysis, testing for the presence of distinct phenotypic modules along the dorsal axis, indicates that plastic responses in the nuchal area are independent of changes in other body parts: there is strong statistical evidence for either two (A-E) or three $(\mathrm{F}-\mathrm{H})$ independent dorsal modules separating areas of largest plasticity (i.e., head area) and the remaining dorsal areas along the carapace. The extent of modularity is described by a CR coefficient in proposed modules compared against randomly assigned groups of landmarks using a random permutation procedure. O cluster: 
A

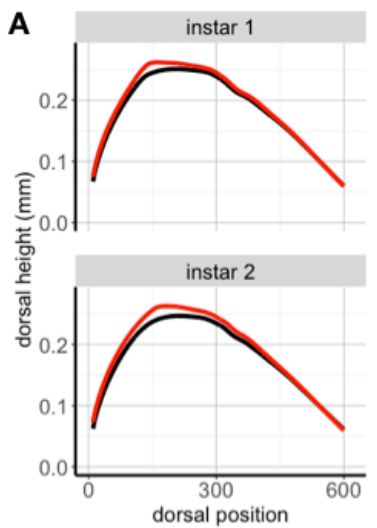

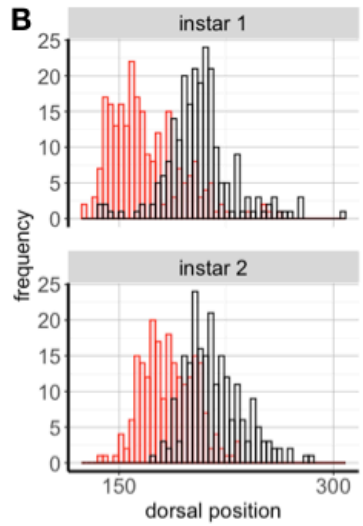
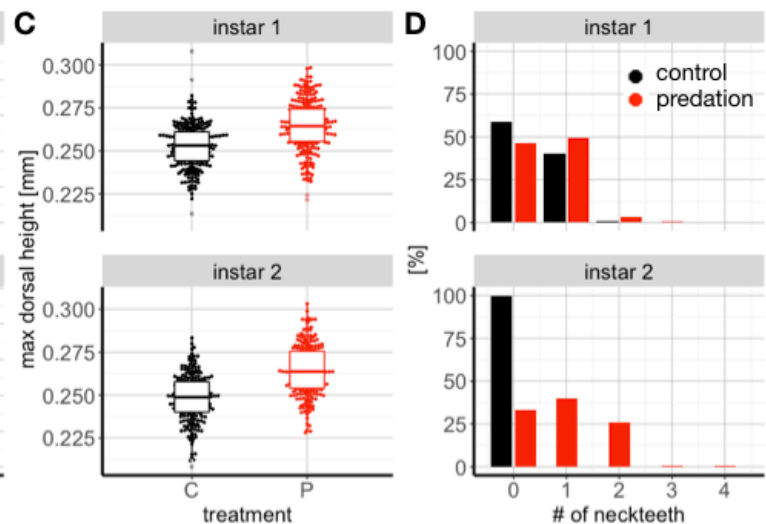
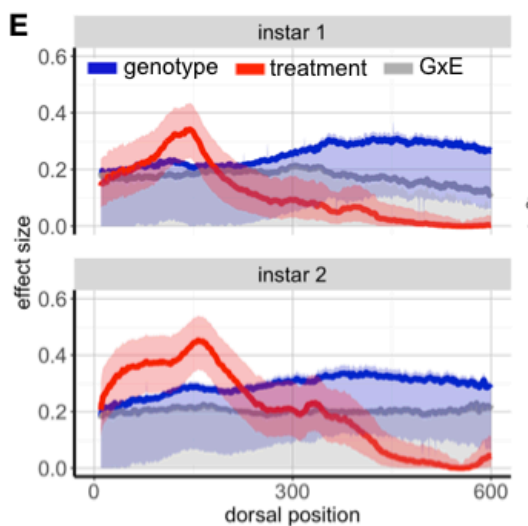

$\mathbf{F} \quad$ instar 1

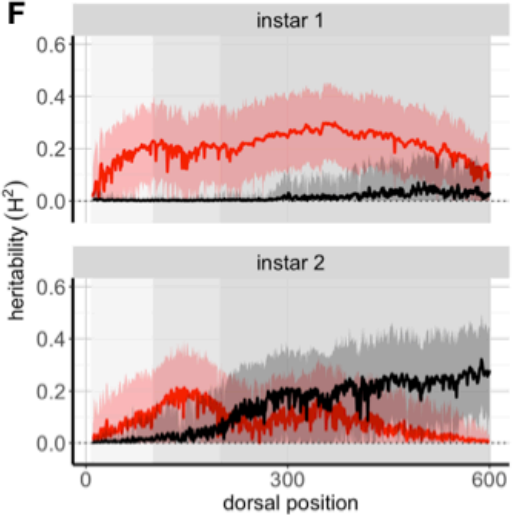

Supplemental Figure 5. Effects of predation risk on morphological changes in genetically similar strains. (A) Risk of predation induces plastic responses, with strongest phenotypic changes observed in the nuchal area (around dorsal positions 100-250). (B,C) In response to predation, maximum dorsal height increases and shift towards anterior head regions. (D) In addition, the number of neckteeth increases und predation risk. Notably, variation in morphological changes within genetically identical clones is as pronounced as that observed among genetically unique clones (see Fig. 2). (E) Effect sizes from analyses of variance along the dorsal shape reveal distinctive patterns of treatment (i.e., predation risk, red line), genotype (blue line), and GxE (grey line) effects on morphological changes. Shaded areas indicate upper (0.95) and lower $(0.05)$ confidence intervals. (F) Broad sense heritability estimates of dorsal height vary along the dorsal axis in response to control conditions (black line) and predation risk (red line) in genetically similar 590 clones. Grey rectangles highlight morphological independent shape modules, separating head and posterior body areas (see Suppl. Fig. 4). 


\section{Supplemental Methods}

High-throughput image analysis: We assessed phenotypic changes using an

594 automated image analysis pipeline hereafter referred to as DAPCHA, using Python

595 version 2.7. First, we identified the eye as a common landmark for each individual

596 image: we first identified the center of the eye by calculating the mean of the

597 darkest $2.5 \%$ of all pixels in the image. The rest of the pixels comprising the eye

598 were identified using a 4-way flood-fill style algorithm on the image after filtering 599 with a Canny edge detector implemented in OpenCV [84], Gaussian blur sigma =

6000.5 , Canny edge filter with lower threshold $=0$, and higher threshold $=50$ ). Next, 601 we identified the center pixel of the animal by taking the mean of all edge pixels 602 produced by a Canny edge filter (lower threshold $=0$, higher threshold $=50$ ) 603 applied to a Gaussian blurred image (sigma =1.25). Then, the tip of the tail was 604 approximated by computing the dot product between the animal center - eye center 605 vector and every animal center - edge pixel vector. The maximum dot product of 606 these were considered the tail tip, since the tail tip should lie the furthest away from 607 the eye. To estimate the animal position within the image, an ellipse was fitted to 608 the edge pixels, providing the center, major and minor axes and orientation of the 609 body of the animal. The major axis vertices were assigned anatomical directions 610 (anterior and posterior) by comparing their locations relative to the eye center. 611 Similarly, the minor axis vertices locations were compared to the tail tip. The 612 ventral and dorsal-most eye points were found by translating the minor axes 613 vectors to originate at the center of the eye and finding the shortest distance 614 between all eye points and the terminal point of the vector. Next, the base of the 615 tail was estimated by finding where the tail spine begins to thicken and joins the 616 bottom of the main carapace. A line segment was drawn between the tail tip 617 towards the eye and divided into 100 points. Starting at the tail tip, a second line 618 segment was drawn between each of the 100 points and a point perpendicular to 619 the eye-tail line. Along this line, the outermost edge pixel was identified (i.e., the 620 edge of the tail spine). If this pixel was above a distance threshold $\left(1 / 5^{\text {th }}\right.$ of the 621 distance between the starting and ending points of the line segment), the search 
622 was terminated, and the pixel was designated as the tail point. The line segment

623 with this tail point edge pixel was extended dorsally, and the furthest edge pixel on

624 that side was designated as the dorsal tail point (i.e., the base of the tail on the

625 dorsal side of the animal). To locate the head, a line segment was drawn between

626 the new tail point and the dorsal eye point. This line segment was then extended

627 until no more edge pixels were found. The last edge pixel was designated as the

628 head point. Using these landmarks, we calculated animal length, animal area, eye 629 area, tail spine length.

630 After landmark detection, the dorsal edge of the carapace was traced by 631 connecting edge pixels between checkpoints along the dorsal edge. These 632 checkpoints were determined by drawing a line segment between the eye and tail, 633 dividing this segment into ten points, and then finding the most dorsal edge pixel 634 along the line perpendicular to the eye-tail line containing these ten points. Due to 635 noise and variability, some of these checkpoints may fall far from the actual dorsal 636 edge of the carapace. These wayward points were pruned from the checkpoint list 637 by calculating the distance of checkpoint from the line drawn between its 638 neighboring checkpoints, discarding it if too far away from the line. After 639 checkpoints were determined, the dorsal edge was traced in segments between 640 checkpoints. Via an iterative search process, one checkpoint per each segment 641 was designated as the target, and one as the current point. Relative to the current 642 pixel, the next pixel should be the one that was both dorsally and posteriorly 643 located and further away from previously traced points. To calculate a position 644 score, all of the edge pixels around the current point within a small neighborhood 645 were indexed and a score was calculated by adding the dot product between the 646 index and three different vectors: a vector between the current point and the target 647 point, a vector between the last current point and the current point, and the vector 648 starting at the animal center and ending at the current point. The edge pixel 649 corresponding the maximum score was labeled as a dorsal edge and then set as 650 the current point. This procedure continued until the target point was reached. 651 Finally, all of the dorsal edge points from each segment were concatenated 652 together. In order to account for differences in number of pixels due to size 
653 variability among animals, we equalized the number of points between dorsal edge 654 traces: we implemented a greedy algorithm using linear interpolation to add points 655 to the dorsal edge until a set target number of points was reached at 700 . Dorsal 656 edge detection allowed estimation of morphological changes along the entire 657 dorsal shape axis, later referred to as dorsal height.

658 Pixel-to-mm extraction from micrometer images: Each slide micrometer image 659 was cropped to include only the scale line. This was done by automatically 660 detecting the dark edges of the micrometer ring and cropping them out. Next, we 661 applied contrast limited adaptive histogram equalization [84] to increase the 662 contrast of the scale against the brightfield background. We then applied a Canny 663 edge filter [84] (lower threshold=0, higher threshold=175) to the high contrast 664 image and used a Hough [84] line detector to find the largest line in the image (i.e., 665 the cross bar in the scale line). Next, we extracted the intensity of the image along 666 a line parallel to the cross bar, drawn along the tick marks in the scale line. Finally, 667 we applied a Fourier transform to the coordinates of the intensity peaks along this 668 line to measure the frequency of the tick marks. This frequency corresponds to the 669 inverse pixel-to-mm ratio divided by a factor of 40 . 


\section{References}

671 1. Scheiner, S.M., Genetics and evolution of phenotypic plasticity. Annual review of

672 ecology and systematics, 1993. 24(1): p. 35-68.

673 2. Via, S., et al., Adaptive phenotypic plasticity: consensus and controversy. Trends

674

675 Ecol Evol, 1995. 10(5): p. 212-7.

3. Ghalambor, C.K., et al., Adaptive versus non-adaptive phenotypic plasticity and the potential for contemporary adaptation in new environments. Functional ecology, 2007. 21(3): p. 394-407.

4. King, J.G. and J.D. Hadfield, The evolution of phenotypic plasticity when environments fluctuate in time and space. Evolution Letters, 2019. 3(1): p. 15-27.

5. Newman, R.A., Genetic variation for phenotypic plasticity in the larval life history of spadefoot toads (Scaphiopus couchii). Evolution, 1994. 48(6): p. 17731785.

6. Nussey, D.H., et al., Selection on heritable phenotypic plasticity in a wild bird population. Science, 2005. 310(5746): p. 304-306.

7. Scheiner, S., Selection experiments and the study of phenotypic plasticity 1. Journal of Evolutionary Biology, 2002. 15(6): p. 889-898.

8. Reger, J., et al., Predation drives local adaptation of phenotypic plasticity. Nature Ecology \& Evolution, 2018. 2(1): p. 100-107.

9. Sommer, R.J., Phenotypic plasticity: from theory and genetics to current and future challenges. Genetics, 2020. 215(1): p. 1-13.

10. Brakefield, P.M. and N. Reitsma, Phenotypic plasticity, seasonal climate and the population biology of Bicyclus butterflies(Satyridae) in Malawi. Ecological Entomology, 1991. 16(3): p. 291-303.

11. Rountree, D. and H. Nijhout, Hormonal control of a seasonal polyphenism in Precis coenia (Lepidoptera: Nymphalidae). Journal of Insect Physiology, 1995. 41(11): p. 987-992.

12. Scheiner, S.M. and R.D. Holt, The genetics of phenotypic plasticity. X. Variation versus uncertainty. Ecology and evolution, 2012. 2(4): p. 751-767.

13. Bonamour, S., et al., Phenotypic plasticity in response to climate change: the importance of cue variation. Philosophical Transactions of the Royal Society B, 2019. 374(1768): p. 20180178.

14. Fox, R.J., et al., Beyond buying time: the role of plasticity in phenotypic adaptation to rapid environmental change. 2019, The Royal Society.

15. Auld, J.R., A.A. Agrawal, and R.A. Relyea, Re-evaluating the costs and limits of adaptive phenotypic plasticity. Proceedings of the Royal Society B: Biological Sciences, 2010. 277(1681): p. 503-511.

16. Murren, C.J., et al., Constraints on the evolution of phenotypic plasticity: limits and costs of phenotype and plasticity. Heredity, 2015. 115(4): p. 293-301.

17. Yampolsky, L.Y., T.M. Schaer, and D. Ebert, Adaptive phenotypic plasticity and local adaptation for temperature tolerance in freshwater zooplankton. Proceedings of the Royal Society B: Biological Sciences, 2014. 281(1776): p. 20132744.

18. Schmid, M. and F. Guillaume, The role of phenotypic plasticity on population differentiation. Heredity, 2017. 119(4): p. 214-225. 
715 19. Charlesworth, B., R. Lande, and M. Slatkin, A neo-Darwinian commentary on

716

717

718

719

720

721

722

723

724

725

726

727

728

729

730

731

732

733

734

735

736

737

738

739

740

741

742

743

744

745

746

747

748

749

750

751

752

753

754

755

756

757

758

759

macroevolution. Evolution, 1982. 36(3): p. 474-498.

20. Lynch, M., The rate of morphological evolution in mammals from the standpoint of the neutral expectation. The American Naturalist, 1990. 136(6): p. 727-741.

21. Haller, B.C. and A.P. Hendry, Solving the paradox of stasis: squashed stabilizing selection and the limits of detection. Evolution, 2014. 68(2): p. 483-500.

22. Siepielski, A.M., et al., Differences in the temporal dynamics of phenotypic selection among fitness components in the wild. Proceedings of the Royal Society B: Biological Sciences, 2011. 278(1711): p. 1572-1580.

23. Hansen, T.F., Stabilizing selection and the comparative analysis of adaptation. Evolution, 1997. 51(5): p. 1341-1351.

24. Kingsolver, J.G. and S.E. Diamond, Phenotypic selection in natural populations: what limits directional selection? The American Naturalist, 2011. 177(3): p. 346357.

25. Noble, D.W., R. Radersma, and T. Uller, Plastic responses to novel environments are biased towards phenotype dimensions with high additive genetic variation. Proceedings of the National Academy of Sciences, 2019. 116(27): p. 1345213461.

26. Draghi, J.A. and M.C. Whitlock, Phenotypic plasticity facilitates mutational variance, genetic variance, and evolvability along the major axis of environmental variation. Evolution: International Journal of Organic Evolution, 2012. 66(9): p. 2891-2902.

27. Agrawal, A.A., Induced plant defense: evolution of induction and adaptive phenotypic plasticity. Inducible plant defenses against pathogens and herbivores: biochemistry, ecology, and agriculture. American Phytopathological Society Press, St. Paul, MN, 1999: p. 251-268.

28. Tollrian, R., Predator-induced morphological defenses: costs, life history shifts, and maternal effects in Daphnia pulex. Ecology, 1995. 76(6): p. 1691-1705.

29. Hammill, E. and A.P. Beckerman, Reciprocity in predator-prey interactions: exposure to defended prey and predation risk affects intermediate predator life history and morphology. Oecologia, 2010. 163(1): p. 193-202.

30. Beckerman, A.P., et al., A shared mechanism of defense against predators and parasites: chitin regulation and its implications for life-history theory. Ecology and evolution, 2013. 3(15): p. 5119-5126.

31. Barnard-Kubow, K., et al., Polygenic variation in sexual investment across an ephemerality gradient in $<e m>$ Daphnia pulex $</ e m>$. bioRxiv, 2021: $\mathrm{p}$. 2021.06.23.449662.

32. Kondrashov, A.S. and M. Turelli, Deleterious mutations, apparent stabilizing selection and the maintenance of quantitative variation. Genetics, 1992. 132(2): p. 603-618.

33. Richards, C.L., O. Bossdorf, and M. Pigliucci, What role does heritable epigenetic variation play in phenotypic evolution? BioScience, 2010. 60(3): p. 232-237.

34. Latta IV, L.C., et al., The phenotypic effects of spontaneous mutations in different environments. The American Naturalist, 2015. 185(2): p. 243-252. 
760 35. Houle, D., How should we explain variation in the genetic variance of traits?

$761 \quad$ Genetica, 1998. 102: p. 241-253.

762 36. Lind, M.I., et al., The alignment between phenotypic plasticity, the major axis of

37. Laforsch, C. and R. Tollrian, Inducible defenses in multipredator environments: genetic variation and the response to selection. Proceedings of the Royal Society B: Biological Sciences, 2015. 282(1816): p. 20151651.

38. Weiss, L.C., J. Leimann, and R. Tollrian, Predator-induced defences in Daphnia cyclomorphosis in Daphnia cucullata. Ecology, 2004. 85(8): p. 2302-2311.

38. Weiss, L.C., J. Leimann, and R. Tollrian, Predator-induced defences in Daphnia
longicephala: location of kairomone receptors and timeline of sensitive phases to trait formation. Journal of Experimental Biology, 2015. 218(18): p. 2918-2926.

39. Lande, R., Adaptation to an extraordinary environment by evolution of phenotypic plasticity and genetic assimilation. Journal of Evolutionary Biology, 2009. 22(7): p. 1435-1446.

40. Via, S. and R. Lande, Genotype-environment interaction and the evolution of phenotypic plasticity. Evolution, 1985. 39(3): p. 505-522.

41. Kvist, J., et al., Temperature treatments during larval development reveal extensive heritable and plastic variation in gene expression and life history traits. Molecular Ecology, 2013. 22(3): p. 602-619.

42. Charlesworth, B., Causes of natural variation in fitness: evidence from studies of Drosophila populations. Proceedings of the National Academy of Sciences, 2015. 112(6): p. 1662-1669.

43. Muschick, M., et al., Adaptive phenotypic plasticity in the Midas cichlid fish pharyngeal jaw and its relevance in adaptive radiation. BMC Evolutionary Biology, 2011. 11(1): p. 1-12.

44. Salzburger, W., Understanding explosive diversification through cichlid fish genomics. Nature Reviews Genetics, 2018. 19(11): p. 705-717.

45. Halligan, D.L. and P.D. Keightley, Spontaneous mutation accumulation studies in evolutionary genetics. Annual Review of Ecology, Evolution, and Systematics, 2009. 40: p. 151-172.

46. Houle, D., B. Morikawa, and M. Lynch, Comparing mutational variabilities. Genetics, 1996. 143(3): p. 1467-1483.

47. Eberle, S., et al., Hierarchical assessment of mutation properties in Daphnia magna. G3: Genes, Genomes, Genetics, 2018. 8(11): p. 3481-3487.

48. Stenseth, N.C., et al., Ecological effects of climate fluctuations. Science, 2002. 297(5585): p. 1292-1296.

49. Burgmer, T., H. Hillebrand, and M. Pfenninger, Effects of climate-driven temperature changes on the diversity of freshwater macroinvertebrates. Oecologia, 2007. 151(1): p. 93-103.

50. Yan, N.D., et al., Long-term trends in zooplankton of Dorset, Ontario, lakes: the probable interactive effects of changes in $\mathrm{pH}$, total phosphorus, dissolved organic carbon, and predators. Canadian Journal of Fisheries and Aquatic Sciences, 2008. 65(5): p. 862-877.

51. Reed, T.E., D.E. Schindler, and R.S. Waples, Interacting effects of phenotypic plasticity and evolution on population persistence in a changing climate. 
805

52. ASTM, Standard Guide for Conducting Acute Toxicity Tests with Fishes, Macroinvertebrates, and Amphibians. E729-88a. American Society for Testing and Materials, Philadelphia, PA. 20 pp. 1988.

53. Baym, M., et al., Inexpensive multiplexed library preparation for megabase-sized genomes. PloS one, 2015. 10(5): p. e0128036.

54. Bolger, A.M., M. Lohse, and B. Usadel, Trimmomatic: a flexible trimmer for Illumina sequence data. Bioinformatics, 2014. 30(15): p. 2114-2120.

55. Zhang, J., et al., PEAR: a fast and accurate Illumina Paired-End reAd mergeR. Bioinformatics, 2014. 30(5): p. 614-620.

56. Li, H., Aligning sequence reads, clone sequences and assembly contigs with BWA-MEM. arXiv preprint arXiv:1303.3997, 2013.

57. Toolkit, P., Broad institute, GitHub repository. See http://broadinstitute. github. io/picard, 2019.

58. McKenna, A., et al., The Genome Analysis Toolkit: a MapReduce framework for analyzing next-generation DNA sequencing data. Genome research, 2010. 20(9): p. 1297-1303.

59. Poplin, R., et al., Scaling accurate genetic variant discovery to tens of thousands of samples. BioRxiv, 2018: p. 201178.

60. Zheng, X., et al., A high-performance computing toolset for relatedness and principal component analysis of SNP data. Bioinformatics, 2012. 28(24): p. 33263328.

61. Manichaikul, A., et al., Robust relationship inference in genome-wide association studies. Bioinformatics, 2010. 26(22): p. 2867-2873.

62. Beckerman, A.P., G.M. Rodgers, and S.R. Dennis, The reaction norm of size and age at maturity under multiple predator risk. Journal of Animal Ecology, 2010. 79(5): p. 1069-1076.

63. Naraki, Y., C. Hiruta, and S. Tochinai, Identification of the precise kairomonesensitive period and histological characterization of necktooth formation in predator-induced polyphenism in Daphnia pulex. Zoological science, 2013. 30(8): p. 619-625.

64. Schneider, C.A., W.S. Rasband, and K.W. Eliceiri, NIH Image to ImageJ: 25 years of image analysis. Nature methods, 2012. 9(7): p. 671-675.

65. Schindelin, J., et al., Fiji: an open-source platform for biological-image analysis. Nature methods, 2012. 9(7): p. 676-682.

66. Scrucca, L., et al., mclust 5: clustering, classification and density estimation using Gaussian finite mixture models. The R journal, 2016. 8(1): p. 289.

67. Fox, J. and S. Weisberg, An R companion to applied regression. 2018: Sage publications.

68. Ben-Shachar, M.S., D. Lüdecke, and D. Makowski, effectsize: Estimation of Effect Size Indices and Standardized Parameters. Journal of Open Source Software, 2020. 5(56): p. 2815.

69. Collyer, M.L. and D.C. Adams, Phenotypic trajectory analysis: comparison of shape change patterns in evolution and ecology. Hystrix, 2013. 24(1): p. 75.

70. Collyer, M., D. Adams, and M.M. Collyer, Package 'RRPP'. 2021. 
71. Collyer, M.L. and D.C. Adams, RRPP: An r package for fitting linear models to high-dimensional data using residual randomization. Methods in Ecology and Evolution, 2018.9(7): p. 1772-1779.

72. Adams, D.C., et al., Geomorph: Software for geometric morphometric analyses. 2016.

Smirnov, P., M.P. Smirnov, and M. Suggests, Package 'robcor' 2014.

855 74. Hadfield, J.D., MCMC methods for multi-response generalized linear mixed models: the MCMCglmm R package. Journal of statistical software, 2010. 33(2): p. 1-22.

75. Yang, J., et al., GCTA: a tool for genome-wide complex trait analysis. The American Journal of Human Genetics, 2011. 88(1): p. 76-82.

76. Core Team, R., $R$ : A language and environment for statistical computing. $R$ Foundation for Statistical Computing. Vienna, Austria: URL https://www. Rproject. org/.[Google Scholar], 2017.

77. Villanueva, R., Z. Chen, and H. Wickham, ggplot2: elegant graphics for data analysis using the grammar of graphics. 2016, Springer-Verlag, New York, NY.

78. Wilke, C., cowplot: streamlined plot theme and plot annotations for 'ggplot 2'. $R$ package version 0.9. 2; 2017. URL https://CRAN. R-project. org/package= cowplot, 2020.

79. Srinivasan, M.D.a.A., data.table: Extension of 'data.frame`. R package version 1.14.0. https://CRAN.R-project.org/package=data.table. 2021.

80. Weston, M.a.S., foreach: Provides Foreach Looping Construct. R package version 1.5.1. https://CRAN.R-project.org/package=foreach. 2020.

81. Weston, R.A.a.S., doMC: Foreach Parallel Adaptor for 'parallel'. R package version 1.3.7. https://CRAN.R-project.org/package=doMC. 2020.

82. Clarke, E. and S. Sherrill-Mix, Ggbeeswarm: Categorical scatter (violin point) plots. R package version 0.6. 0. Retrieved from https://CRAN. R-project. org, 2017.

880

881

83. Garnier, S., et al., viridis: Default color maps from 'matplotlib'. $R$ package version 0.5. 1. CRAN: the Comprehensive R Archive Network.[Google Scholar], 2018.

84. Bradski, G., The opencv library. Dr Dobb's J. Software Tools, 2000. 25: p. 120125. 